\title{
Multi-wavelength study of a new sample of blue compact dwarf galaxies
}

\section{Optical multi-pupil spectroscopy of 18 objects ${ }^{\star}$}

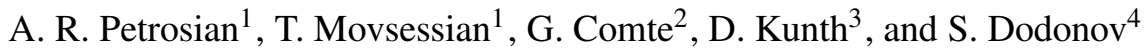 \\ ${ }^{1}$ Byurakan Astrophysical Observatory and Isaac Newton Institute of Chile, Armenian Branch, 378443 Byurakan, Armenia \\ 2 Observatoire Astronomique de Marseille-Provence and Laboratoire d'Astrophysique de Marseille, 2 Place Le Verrier, \\ 13248 Marseille Cedex 04, France \\ 3 Institut d'Astrophysique de Paris, 98bis Boulevard Arago, 75014 Paris, France \\ ${ }^{4}$ Special Astrophysical Observatory, Nizhnij Arkhyz, Karachai-Circassian Republic 357147, Russia
}

Received 18 January 2001 / Accepted 24 May 2002

\begin{abstract}
We present the multi-pupil integral field spectroscopy of 18 Blue Compact Dwarf Galaxies performed at the $6 \mathrm{~m}$ telescope of the SAO (Russia). We produce an atlas of the $\mathrm{H} \alpha$, [OIII], [SII] emission-line intensity distributions, the excitation parameter $([\mathrm{OIII}] / \mathrm{H} \beta$ ratio), the continuum maps in blue and red and the velocity fields as derived from the $2 \mathrm{D}$ spectra. We provide detailed individual descriptions of the objects with a table of their spectroscopic properties.

From the atlas and the velocity maps, we have classified the sample according to the following scheme:

- Nine objects show spatially coincident distributions with respect to their emission lines, excitation parameter and the continuum. In this case, these galaxies are repeating star-formation sequences at the same spatial location. Mrk 1416 also belongs to this class of BCDG but has a secondary site of star formation.

- Eight objects in which the distributions in emission lines brightness, excitation parameter and in continuum strength differ from each other at various degrees. These objects are galaxies with several sites of star formation of various ages and spatial locations. They can be ideal templates for testing the models of star formation.

- From the 2-D [SII] emission-line distribution we find a superbubble structure in one object and possibly in 2 others.

- Ten objects show an overall quite regular radial velocity gradient. On the other hand, in 8 objects, the kinematical major axis of this regular velocity field does not align with the photometric major axis, suggesting dynamical disturbances, gas/star decoupling and/or instabilities.

- Six objects have irregular velocity fields, implying that the rotation of a gaseous disk is not their dominant dynamical signature. - SBS $1723+565 B$ is not a dwarf galaxy but a giant irregular galaxy or an interacting system experiencing a high star formation rate.
\end{abstract}

Key words. galaxies: dwarf - galaxies: starburst - galaxies: kinematics and dynamics

\section{Introduction}

Star formation parameters such as the shape of the initial mass function (IMF) and the star formation rates $(S F R \mathrm{~s})$, are crucial in understanding the evolution of galaxies (Silk 1995). Data from different spectral windows in both the continuum and the spectral lines allow one to estimate their characteristics.

It is well established that excitation parameters such as the $[\mathrm{OIII}] / \mathrm{H} \beta$ ratio as well as the Balmer and some forbidden emission-lines luminosities are indicators of the current instantaneous $S F R$ in galaxies (e.g. Lequeux 1980; Güsten \& Mezger 1983; Gallagher et al. 1984; Hunter \& Gallagher 1986),

\footnotetext{
Send offprint requests to: D. Kunth, e-mail: kunth@iap.fr

* Based on observations performed with the Special Astrophysical Observatory (Russia) $6 \mathrm{~m}$. Telescope.
}

at least with respect to the massive stars formed over the last tens of Myrs. The $B$-band luminosity traces the $S F R$ averaged over a longer duration i.e. the lifetime of stars dominating the blue light $\left(0.4-6 \times 10^{9}\right.$ yr) (Gallagher et al. 1984; Thronson $\&$ Telesco 1986). Finally, the red continuum is a tracer of the older, evolved stellar population. Hence, mapping in suitable filters and continuum bands can provide clues about current, present and past formation history in systems too distant to be resolved or in which direct color magnitude diagrams cannot be derived by observation.

The triggering mechanism of star formation (hereafter SF) in galaxies is another critical topic. In spiral galaxies it is widely accepted that strong density waves trigger SF. In irregular galaxies as well as Blue Compact Dwarf Galaxies (BCDGs) 
density waves seem absent and other possible SF triggering mechanisms should be considered, such as galaxy-galaxy interactions (e.g. Kennicutt et al. 1987; Brinks 1990; Noguchi 1991), infall of gas clouds from an extended HI envelope surrounding the central region (Saito et al. 1992), chimney model (Li \& Ikeuchi 1989) or stochastic self-propagating SF (Gerola et al. 1980). Besides, the structure of the velocity field itself can lead to new ideas about SF and its triggering mechanism (e.g. Martin 1996; Petrosian et al. 1997; van Zee et al. 1998; Östlin et al. 1999).

It is also useful to study the spatial distribution of the shock-heated lines of [SII] and [OI]. They are tracers of galactic winds powered by $\mathrm{SNe}$ and play an important role in the heavy elements mixing scenario (Matteucci \& Tosi 1985; Marconi et al. 1994; Roy \& Kunth 1995). Finally, it has also been suggested that, even in an extreme metal-poor starburster such as IZw18, a low-rate continuous SF could have proceeded over very long timescales (Legrand 2000; Legrand et al. 2000).

BCDGs have long been recognized as clues to our understanding of star-formation processes and galaxian evolution. They are among the best targets allowing to attack the above stressed problems. Evidence for this view is reienforced by their blue colours (in average $B-V=0.2$ and $U-B=-0.6$ ), high ratios of $\mathrm{HI}$ to total mass (e.g. Thuan \& Martin 1981; Viallefond et al. 1987), low heavy element abundances (e.g. Searle \& Sargent 1972; Thuan et al. 1995, but see Östlin \& Kunth 2001), and a morphology dominated by the presence of one or several high surface brightness giant star-forming regions that outshine an older underlying population of stars (Loose \& Thuan 1986a; Kunth et al. 1988; Hunter \& Thronson 1995; Doublier et al. 1999). The dynamics of such galaxies are determined by turbulent motions on small scales (Terlevich \& Melnick 1981; Gallagher \& Hunter 1983; Skillman \& Kennicutt 1993 ; Lequeux et al. 1995; Martin 1996; Petrosian et al. 1997) and little rotation (Arkhipova et al. 1987; Thuan et al. 1987) often indiscernible from pure solidbody rotation (Tomita et al. 1993; Petrosian et al. 1997; Östlin et al. 1999). Although a large amount of information has been hitherto collected about BCDGs some tantalizing problems still need clarification:

- Is the shape of their IMF universal (Salpeter-type), or does it depend on the structure of the object (see Mas-Hesse \& Kunth 1999)?

- What triggering mechanism(s) of star formation is (are) at work?

- Are they chemically evolving as isolated systems (closedbox models)?

- What are their progenitors? Is it possible to observe postphases? What is the appearance and photometric/spectroscopic properties of these post-phases?

We have undertaken a multi-wavelength study of a new sample of BCDGs in order to improve the database susceptible to provide answers to the above mentioned questions. The sample was built with objects from the First and Second Byurakan, Case, Kiso, Wasilewski and University of Michigan surveys. Papers I and II of the series describe $B$ and $R$ surface photometry of the sample (Doublier et al. 1997, 1999). They have provided evidence for the common occurrence of an evolved red population giving the bulk of the background light in the host galaxies of most BCDGs. This has been confirmed on a reduced sample by surface photometry in $J H K$ colors (Doublier et al. 1998). Paper III discusses HI observations (Comte et al. 1999).

In the present paper, multi-pupil spectroscopic observations performed at the $6 \mathrm{~m}$ telescope of SAO (Russia) are presented. We describe the morphology of 18 BCDGs as they appear in high and low ionization emission lines and in blue and red continuum. The results are interpreted with respect to the SF history of the objects. We study their velocity fields and conclude about possible triggering mechanisms. We report on the spatial distribution of some diagnostic emission lines that give clues to chemical evolution of the objects.

The paper is organized as follows: we describe the multipupil observations and data reduction in Sect. 2. Results are presented in Sect. 3, with individual comments on each galaxy while they are further discussed in Sect. 4. Conclusions are summarised in Sect. 5.

We shall adopt a Hubble constant $H_{\mathrm{o}}=75 \mathrm{~km} \mathrm{~s}^{-1} \mathrm{Mpc}^{-1}$.

\section{Observations and data reduction}

Observations have been performed at the prime focus of the $6 \mathrm{~m}$. telescope of Special Astrophysical Observatory (SAO, Zelenchuk, Russia) with the multi-pupil panoramic spectrophotometer, providing bidimensional spectroscopy by means of a microlenses array dissector (Afanasiev et al. 1990) along an optical scheme basically similar to that of the Tiger spectrograph (Courtès 1982; Bacon et al. 1995). The journal of observations is given in Table 1 . The spectrophotometer was constructed at the SAO (e.g. Sil'chenko 1997). A $9 \times 11$, square microlenses array was used giving a scale of $1.3 \times 1.3$ arcsec for each lens unit. Two spectral regions have been observed: bluegreen (4800-5200 $)$ ) and red (6300-6700 $)$ with a reciprocal dispersion of about $1.5 \AA$ per pixel (spectral resolution about $7 \AA$ ). The wavelength scale was determined from $\mathrm{Ne}+\mathrm{Xe}+\mathrm{Ar}$ calibration lamp exposures and the sensitivity response functions were derived from classical standard stars (Massey et al. 1988). Ninety nine spectra are simultaneously obtained on each frame. The detectors were a $512 \times 512$ pixels photon counting system during the 1994 observing run and a $580 \times 530$ pixels CCD during all the 1995 observing runs. All observations were made in seeing conditions better than $2^{\prime \prime}$.

The data reduction was made in two steps using a software developed at the SAO (Vlasiuk 1993). This involves correction for distortion, pixel-to-pixel sensitivity variations and flatfielding. Individual spectra, corresponding to each microlens, were extracted from the two-dimensional frames. Each spectrum was wavelength scaled and calibrated using standard procedures. The redshifted wavelength uncertainties of emission lines vary from lens to lens and are of the order of 20$30 \mathrm{~km} \mathrm{~s}^{-1}$ in radial velocity units. When averaged over all microlenses, the instrumental profile is well fitted with two arithmetically-added gaussian profiles of $F W H M 194 \mathrm{~km} \mathrm{~s}^{-1}$ and $408 \mathrm{~km} \mathrm{~s}^{-1}$ respectively. Intensity distribution maps have been built from arrays centered onto $\mathrm{H} \beta$, [OIII]5007, $\mathrm{H} \alpha$, 
Table 1. Log of multi-pupil observations.

\begin{tabular}{llll}
\hline \hline Galaxy & Date & $t_{\text {Blue }}(\mathrm{s})$ & $t_{\text {Red }}(\mathrm{s})$ \\
\hline Mrk33 & 21 May 95 & 1200 & 1200 \\
Mrk324 & 29 Aug. 95 & 2400 & 2400 \\
Mrk324 & 09 Sep. 95 & 2400 & 2400 \\
Mrk826 & 26 May 95 & 1200 & 1200 \\
Mrk900 & 29 Aug. 95 & 2400 & 2400 \\
Mrk1416 & 18 Apr. 94 & 2400 & 2400 \\
Mrk1426 & 28 May 95 & 2400 & 2400 \\
Mrk1426 & 28 May 95 & 1200 & 1200 \\
Mrk1434 & 21 May 95 & 2400 & 1800 \\
Mrk1450 & 17 Apr. 94 & 2400 & 2400 \\
Mrk1480 & 23 Feb. 95 & 5400 & - \\
Mrk1499 & 21 May 95 & 2400 & 2400 \\
SBS0136+328 & 01 Sep. 95 & 2400 & 2400 \\
SBS1154+534 & 30 May 95 & 2400 & 2400 \\
SBS1331+493 & 18 Apr. 94 & 1800 & 1800 \\
SBS1400+461 & 29 May 95 & 1800 & 1800 \\
SBS1428+457 & 22 May 95 & 1800 & 1800 \\
SBS1632+579 & 01 Sep. 95 & 3600 & 2400 \\
SBS1707+565 & 30 Aug. 95 & 2400 & 2400 \\
SBS1723+565B & 28 Aug. 95 & 1800 & 2400 \\
\hline
\end{tabular}

[NII]6584 and [SII]6717+6731 as well as those corresponding to the blue and red continuum. The continuum maps were built by selecting several emission-free continuum windows

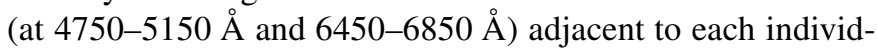
ual spectrum. A polynomial fitting of the continuum level was performed across these windows and the derived continuum model was integrated across a square passband $400 \AA$ wide. From these maps, the $\mathrm{H} \beta$ emission-line equivalent width and emission-line ratios were derived. In some cases, the signalto-noise in $[\mathrm{SII}]$ emission lines was insufficient to allow a meaningful mapping (Mrk1450, Mrk1480, SBS 0136+328, SBS 1331+493 and SBS 1632+579).

The velocity fields were constructed using the strongest emission lines [OIII]5007 and $\mathrm{H} \alpha$.

In addition to the detailed mapping, a "total" spectrum was produced by co-adding all individual spectra in order to study integral properties of the star-forming region. This integral spectrum has been built for any well-separated star-forming region that could be recognized as an individual morphological entity. Therefore, objects that appear to have only one star-forming component will be described by a single line in Table 2, while objects exhibiting several separated star forming components across the observed area will be described by several lines with suffixes $(a, b, c)$. The errors on each observed line ratio have been estimated from the noise in the continuum near both emission lines. The same approach was used to estimate the errors on the equivalent widths of $\mathrm{H} \beta$ line. Aside from SBS $1154+534$, we did not detect underlying $\mathrm{H} \beta$ absorption due to hot main sequence population on the co-added spectra, because of insufficient spectral resolution. Therefore, the $E W \mathrm{~s}$ given in Table 2 were not corrected for this effect. Comparisons with previous spectroscopic results are given in subsection 3.4 below. Some discrepancies on EWs measurements appear in several cases: it should be emphasized that the present co-added spectra result from the integration of line and continuum emission across a rectangular area of some 160 square arc seconds while all preceding spectroscopic investigations used narrow slits. Depending on the exact pointing of the slits, large differences could be expected whether a nebular line emission brightness maximum is coincident or not with the underlying continuum maximum.

\section{Results}

\subsection{Integral and spectroscopic properties}

Except for Mrk 826, Mrk 1416, Mrk 1450 and SBS 1331+493 the spectra of all objects have been flux-calibrated. Table 2 presents integral spectroscopic parameters and is organized as follows:

Column 1: Galaxy name.

Column 2: Heliocentric radial velocities measured from the "total" multi-pupil spectra.

Columns 3-6: [OIII] $(5007+4959) \AA$ to $\mathrm{H} \beta, \mathrm{H} \alpha$ to $\mathrm{H} \beta$, [NII](6584+48) $\AA$ and [SII] $(6717+31) \AA$ to $\mathrm{H} \alpha$ emission lines intensity ratios. The errors are evaluated as indicated in the previous subsection.

Column 7: Equivalent widths of $\mathrm{H} \beta$ line in angströms.

Column 8: Clockwise position angles (PA) of the kinematic major axis of the velocity field (in degrees).

Column 9: Radial velocity gradients expressed in $\mathrm{km} \mathrm{s}^{-1} \mathrm{pc}^{-1}$. Column 10: References to previous work on individual objects.

\subsection{Emission lines and continuum maps}

Maps of several emission lines as well as the red and blue continuum have been built for all observed objects. Figures 1.1 to 1.18 show the [OIII]5007 grey scale images of the sample galaxies with the blue continuum contours overlaid. Figures 2.1 to 2.17 show the $\mathrm{H} \alpha$ grey scale images together with the red continuum contours overlaid. It is not possible to assess an absolute brightness scale on these maps because: i) several objects could not be observed in good atmospheric conditions to derive an accurate absolute flux calibration, ii) for a substantial part of the sample, the sky area covered by the microlens array is smaller than the size of the galaxy. Therefore, the isophotes on these maps are drawn on a relative scale: on each map, the step between two successive isophotes is a constant percentage of the maximum flux value of the map. For most maps, this step is approximatively $10 \%$ of the maximum flux. Specific cases are indicated in the figure captions.

The blue and red continuum were measured in a $400 \AA$ bandwidth as explained above. These maps allow a detailed comparison of the brightness distribution in the continuum (which traces the recent stellar population projected density), with that observed in emission lines, this latter indicating the presence of interstellar gas ionized by massive stars. The local peaks in emission lines brigthness trace the location of the currently (Myrs) and/or very recently (0.4 to several $10^{6}$ yrs) born massive stellar population.

Figures 3.1 to 3.18 present [OIII]/H $\beta$ contour maps with the [OIII]5007 grey scale image overlayed. This ratio is basically 
Table 2. Spectroscopic properties of the observed galaxies.

\begin{tabular}{|c|c|c|c|c|c|c|c|c|c|}
\hline BCDG & $\begin{array}{l}V_{\mathrm{r}} \\
\left(\mathrm{km} \mathrm{s}^{-1}\right)\end{array}$ & {$[\mathrm{OIII}] / \mathrm{H} \beta$} & $\mathrm{H} \alpha / \mathrm{H} \beta$ & {$[\mathrm{NII}] / \mathrm{H} \alpha$} & {$[\mathrm{SII}] / \mathrm{H} \alpha$} & $\begin{array}{l}E W(\mathrm{H} \beta) \\
(\AA)\end{array}$ & $\begin{array}{l}\text { PA } \\
\text { (deg) }\end{array}$ & $\mathrm{d} V$ & $\begin{array}{l}\text { Ref. to } \\
\text { prev. work }\end{array}$ \\
\hline Mrk 33 & $1476 \pm 11$ & $2.87 \pm 0.12$ & $5.33 \pm 0.11$ & $0.17 \pm 0.03$ & $0.17 \pm 0.02$ & $24 \pm 1$ & 180 & 0.118 & $3,6,7,10,13$ \\
\hline Mrk 324 & $1593 \pm 12$ & $4.15 \pm 0.66$ & $4.40 \pm 0.44$ & $0.10 \pm 0.04$ & $0.26 \pm 0.07$ & $5 \pm 1$ & 55 & $0.063:$ & $1,2,8,11$ \\
\hline Mrk 826 & $737 \pm 15$ & $4.83 \pm 1.48$ & & $0.07:$ & $0.18 \pm 0.05$ & $7 \pm 1$ & 70 & 0.543 & \\
\hline Mrk 826a & $743 \pm 13$ & $5.20 \pm 1.32$ & & & $0.18 \pm 0.07$ & $10 \pm 1$ & & & \\
\hline Mrk 826b & $741 \pm 15$ & $3.31 \pm 1.50$ & & & & $17 \pm 2$ & & & \\
\hline Mrk 900 & $1161 \pm 11$ & $3.62 \pm 0.24$ & $3.65 \pm 0.21$ & $0.13 \pm 0.03$ & $0.24 \pm 0.02$ & $10 \pm 1$ & & & 5,11 \\
\hline Mrk 1416 & $2295 \pm 13$ & $6.43 \pm 0.72$ & & $0.03 \pm 0.01$ & $0.09 \pm 0.03$ & $3 \pm 1$ & 270 & 0.080 & 11,12 \\
\hline Mrk 1416a & $2279 \pm 12$ & $7.80 \pm 0.86$ & & & $0.09 \pm 0.03$ & $27 \pm 5$ & & & \\
\hline Mrk $1416 b$ & $2269 \pm 15$ & $6.50 \pm 0.72$ & & & & $10 \pm 4$ & & & \\
\hline Mrk 1426 & $1891 \pm 11$ & $3.58 \pm 0.19$ & $5.40 \pm 0.32$ & $0.12 \pm 0.01$ & $0.37 \pm 0.03$ & $7 \pm 1$ & & & 11,12 \\
\hline Mrk 1426a & $1899 \pm 13$ & $4.97 \pm 0.86$ & $4.12 \pm 0.25$ & $0.05 \pm 0.01$ & $0.43 \pm 0.02$ & $20 \pm 2$ & & & \\
\hline Mrk 1426b & $1865 \pm 15$ & $2.88 \pm 0.15$ & $4.82 \pm 0.29$ & $0.15 \pm 0.01$ & $0.31 \pm 0.03$ & $15 \pm 1$ & & & \\
\hline Mrk $1426 c$ & $1859 \pm 20$ & $2.47:$ & $5.57 \pm 0.33$ & & $0.17:$ & $9 \pm 1$ & & & \\
\hline Mrk 1434 & $2265 \pm 23$ & $7.14 \pm 0.53$ & $3.06 \pm 0.27$ & $0.06 \pm 0.01$ & $0.07 \pm 0.02$ & $65 \pm 5$ & & & 11,12 \\
\hline Mrk 1450 & $984 \pm 10$ & $7.77 \pm 0.82$ & & $0.02 \pm 0.01$ & $0.10 \pm 0.01$ & $11 \pm 1$ & & & 11,12 \\
\hline Mrk 1480 & $1808 \pm 12$ & $6.78 \pm 1.09$ & & & & $14 \pm 1$ & & & 11 \\
\hline Mrk 1499 & $2714 \pm 16$ & $3.39 \pm 0.40$ & $3.12 \pm 0.11$ & $0.10 \pm 0.02$ & $0.41 \pm 0.06$ & $25 \pm 2$ & 35 & 0.115 & 4,11 \\
\hline SBS $0136+328$ & $6244 \pm 31$ & $2.46 \pm 0.90$ & $2.5 \pm 1.1$ & $0.28 \pm 0.11$ & $0.29 \pm 0.13$ & $9 \pm 2$ & 320 & 0.080 & 11 \\
\hline SBS 1154+534 & $1107 \pm 9$ & $3.36 \pm 0.42$ & $3.02 \pm 0.36$ & $0.13 \pm 0.01$ & $0.20 \pm 0.02$ & $12 \pm 1$ & & & \\
\hline SBS $1154+534 a$ & $1107 \pm 12$ & $4.60 \pm 0.58$ & $4.98 \pm 0.60$ & $0.08 \pm 0.01$ & $0.30 \pm 0.03$ & $11 \pm 1$ & & & \\
\hline SBS $1154+534 b$ & $1129 \pm 14$ & $2.68:$ & $3.06 \pm 0.37$ & $0.18 \pm 0.01$ & $0.14 \pm 0.01$ & $11 \pm 1$ & & & \\
\hline SBS 1331+493 & $608 \pm 11$ & $7.46 \pm 0.83$ & & & & $27 \pm 1$ & $255:$ & $0.250:$ & $9,11,12$ \\
\hline SBS $1400+461$ & $2140 \pm 8$ & $2.43 \pm 0.29$ & $2.80 \pm 0.32$ & $0.24:$ & $0.24 \pm 0.01$ & $9 \pm 1$ & 110 & 0.100 & \\
\hline SBS $1428+457$ & $2369 \pm 9$ & $5.09 \pm 0.40$ & $2.85 \pm 0.29$ & $0.08 \pm 0.01$ & $0.14 \pm 0.01$ & $28 \pm 1$ & 150 & 0.070 & 11 \\
\hline SBS $1632+579$ & $5225 \pm 12$ & $4.88 \pm 1.13$ & $2.68 \pm 0.73$ & & & $29 \pm 1$ & & & \\
\hline SBS $1707+565$ & $3373 \pm 6$ & $5.40 \pm 0.65$ & $3.37 \pm 0.43$ & $0.07 \pm 0.01$ & $0.22 \pm 0.02$ & $10 \pm 1$ & 100 & 0.027 & \\
\hline SBS $1723+565 B$ & $8517 \pm 9$ & $5.45 \pm 0.58$ & $2.86 \pm 0.35$ & $0.07 \pm 0.02$ & $0.18 \pm 0.03$ & $24 \pm 2$ & 318 & 0.065 & \\
\hline SBS $1723+565 B a$ & $8588 \pm 10$ & $4.86 \pm 0.53$ & $2.81 \pm 0.34$ & & $0.29 \pm 0.03$ & $16 \pm 1$ & & & \\
\hline SBS $1723+565 B b$ & $8496 \pm 10$ & $6.16 \pm 0.67$ & $3.37 \pm 0.40$ & $0.08 \pm 0.02$ & $0.14 \pm 0.03$ & $27 \pm 2$ & & & \\
\hline
\end{tabular}

Notes to Table 2: the velocity gradient $\mathrm{d} V$ is expressed in $\mathrm{km} \mathrm{s}^{-1} \mathrm{pc}^{-1}$.

References to previous work are coded as follows: a) morphology and photometry: 1: Barbieri et al. (1979); 2: Koroviakovskii et al. (1981); 3: Loose \& Thuan (1986b); 4: Moles et al. (1987); 5: Abrahamian \& Saakian (1989); 8: Mazzarella \& Boroson (1993); 10: Papaderos et al. (1996); 11: Doublier et al. (1997); b) spectroscopy: 6: Gallagher \& Hunter (1989); 7: Kennicutt (1992); 9: Thuan et al. (1995); 12: Izotov et al. (1997); 13: Legrand et al. (1997).

an excitation indicator, i.e. of the available fraction of hard ionizing photons of the ionizing star cluster embedded in an extragalactic HII region. It is also a useful empirical oxygen abundance estimator whenever the electron temperature cannot be estimated by direct methods. Across a given BCDG, the spatial scale-length is supposed to be sufficiently small (of the order of a kiloparsec or even much smaller) for abundance fluctuations to be probably not detectable using the sole $[\mathrm{OIII}] / \mathrm{H} \beta$ ratio as indicator, the accuracy of its calibration versus $\log [\mathrm{O} / \mathrm{H}]$ being not better than \pm 0.2 dex (see e.g. Edmunds \& Pagel 1984; Zaritsky 1992; Roy et al. 1996). Moreover, classical HII region models show that, for a given abundance, the dilution factor of the ionized gas has an important influence on the computed excitation ratio (see e.g. Evans \& Dopita 1985). Finally, a considerable sensitivity of this ratio to a possible presence of local dust within the ionized region can be expected.

Shock waves can trigger star-formation (e.g. Combes 1994) and/or also trace advanced merging processes in galaxies (e.g. Lamb et al. 1994). [SII] lines are among the best diagnostics of the shock wave heating in ionized regions (e.g. Shull \& McKee 1979). Hence comparative mapping of the extended ionized regions in $\mathrm{H} \alpha$ and [SII] emission lines can be useful to search for shock wave heating effects in BCDGs (e.g. Schwartz et al. 1993). Figures 4.1 to 4.13 show $\mathrm{H} \alpha$ grey scale images, with the [SII] lines contours overlaid, for the 13 galaxies in which the signal-to-noise in [SII] lines was sufficient.

\subsection{Velocity field}

A detailed study of the velocity field structure of the ionized gas component provides clues to understand the triggering mechanism of star-formation in BCDG. The two bright emission lines $\mathrm{H} \alpha$ and [OIII]5007 have been used to this purpose. Figures 5.1 to 5.18 show iso-velocity lines superimposed on $\mathrm{H} \alpha$ intensity maps, except for Mrk 1480, observed only in the blue spectral range, for which iso-velocity lines are superimposed instead on the [OIII]5007 intensity map. Table 2 gives position angles (PA) of the kinematic major axis and estimates of the radial velocity gradient for galaxies in which a clear overall gradient is observed and supposedly traces ordered large-scale rotation. 


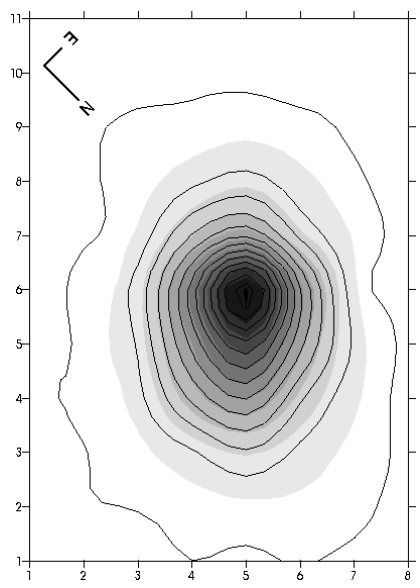

1.1 : Mrk 33

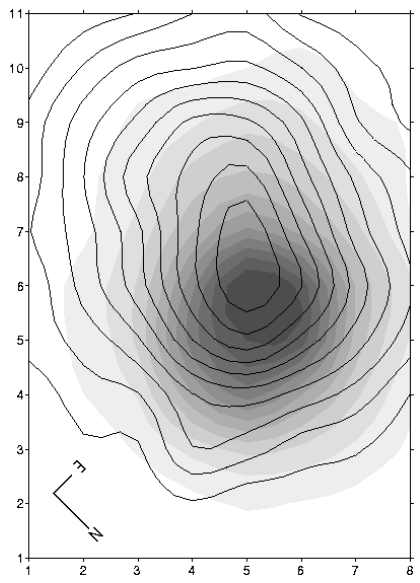

1.4 : Mrk 900

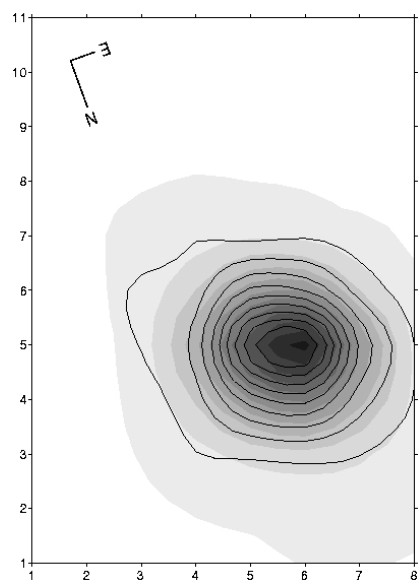

1.7 : Mrk 1434

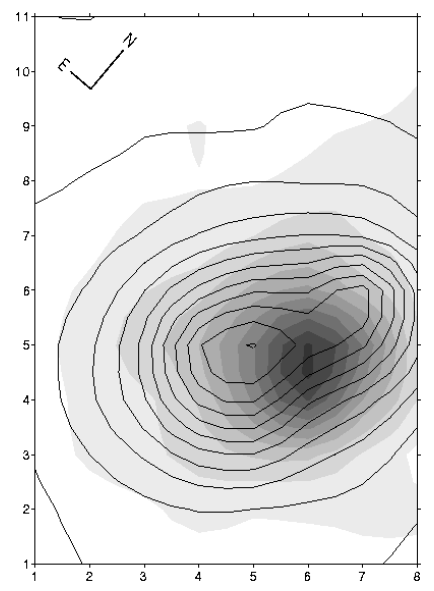

1.2 : Mrk 324

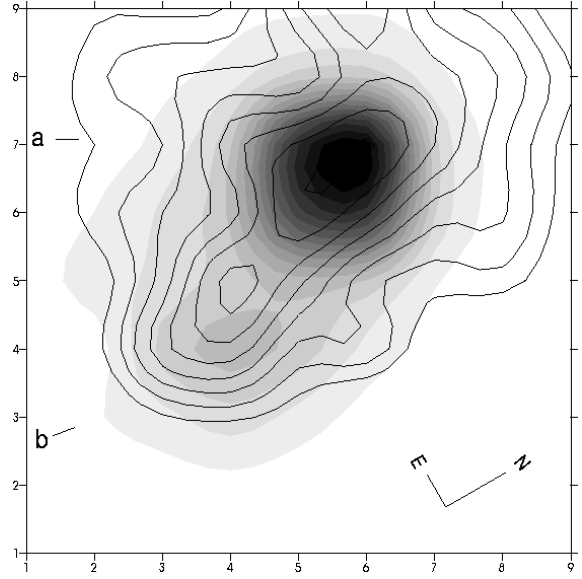

1.5 : Mrk 1416

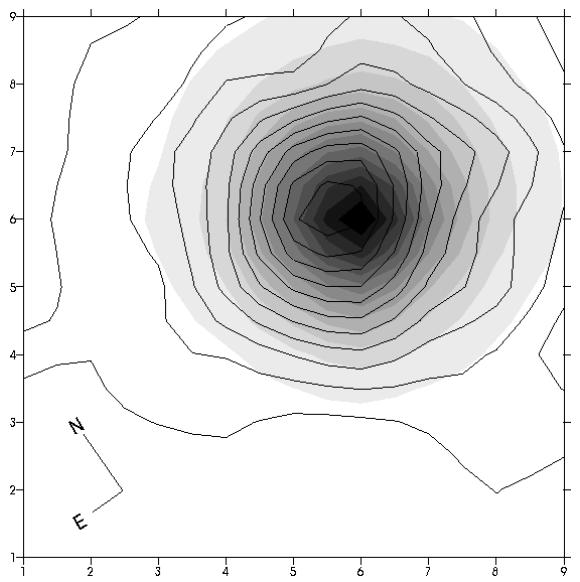

1.8 : Mrk 1450

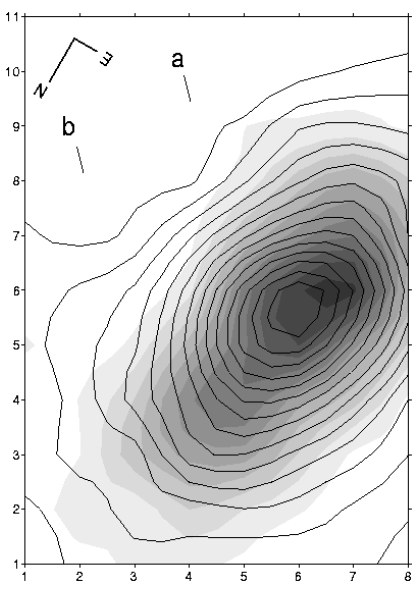

1.3 : Mrk 826

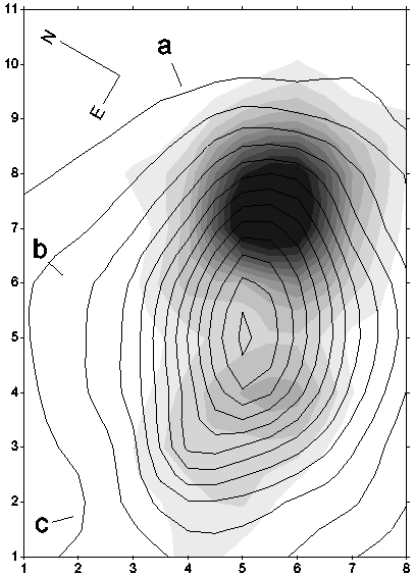

1.6 : Mrk 1426

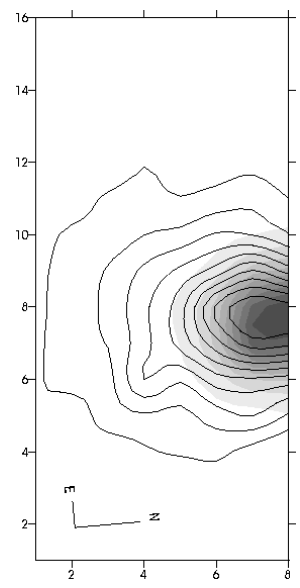

1.9 : Mrk 1480

Fig. 1. Distribution of [OIII] emission line intensity in grey halftone with the distribution of the blue continuum integrated in a bandwidth of $400 \AA$ Averplotted as isophotal contours for the 18 observed galaxies. The brightness scales are relative: i) isophotal step for [OIII]: Mrk 1416, 1426, 1450: approx. 5\% of maximum; SBS 1723+565B: approx. 15\% of maximum; ii) isophotal step for continuum: Mrk 826, 900, 1416, 1450, 1480, SBS $1154+534,1331+493,1400+461,1428+457,1632+579,1707+565$ : approx. $5 \%$ of maximum. The tickmark interval on the frames sides is 1.3 arcsec, the angular extent subtended by a microlens. 


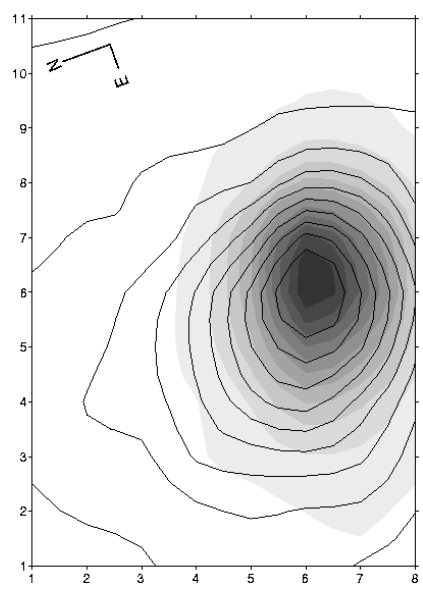

1.10 : Mrk 1499

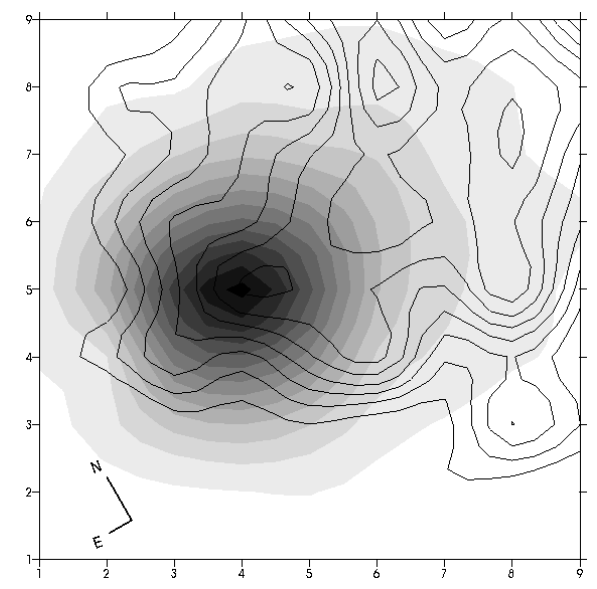

1.13 : SBS 1331+493

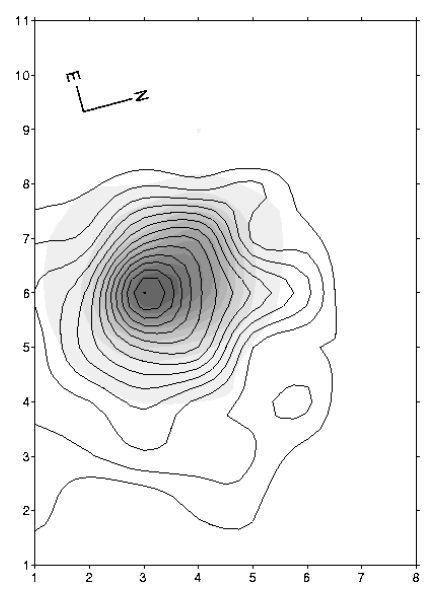

1.16 : SBS 1632+579

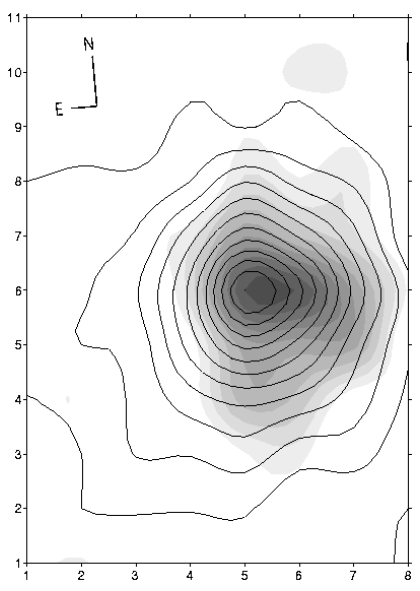

1.11 : SBS 0136+328

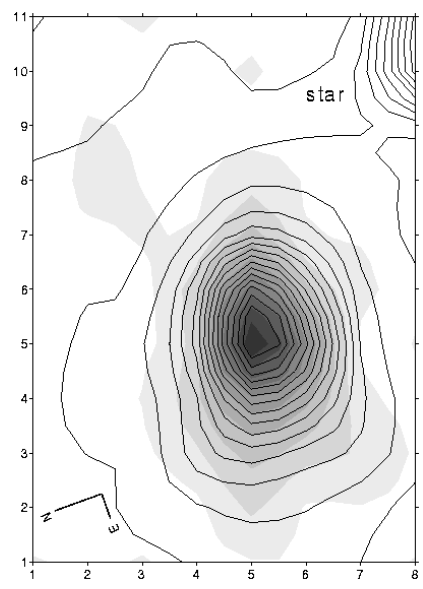

1.14 : SBS 1400+461

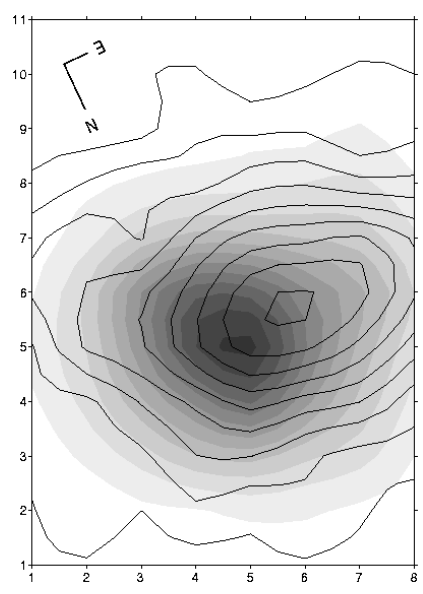

1.17 : SBS 1707+565

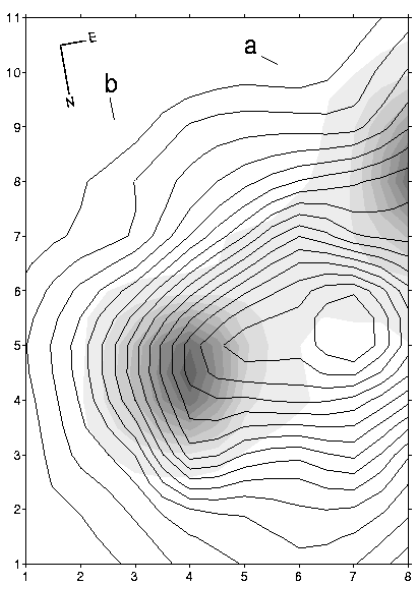

1.12 : SBS 1154+534

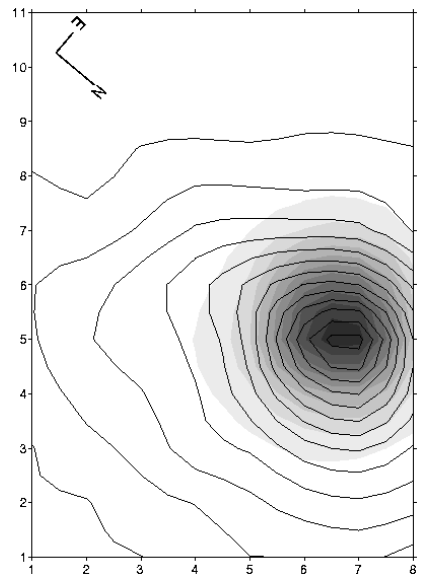

1.15 : SBS 1428+457

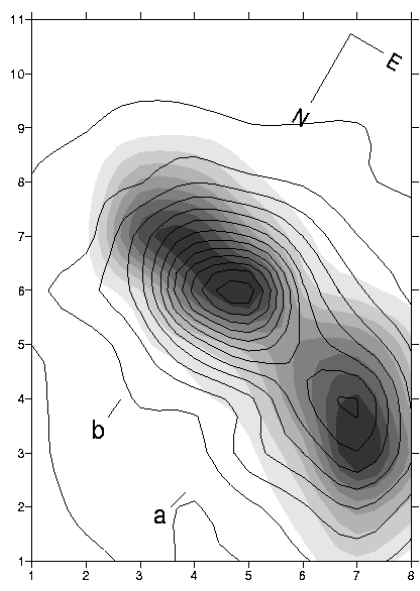

1.18 : SBS 1723+565

Fig. 1. continued. 


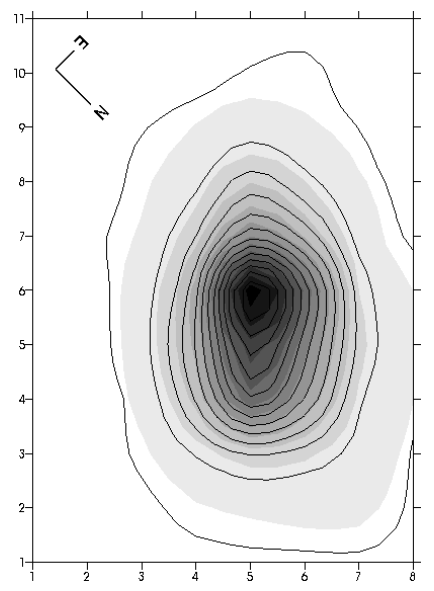

2.1 : Mrk 33

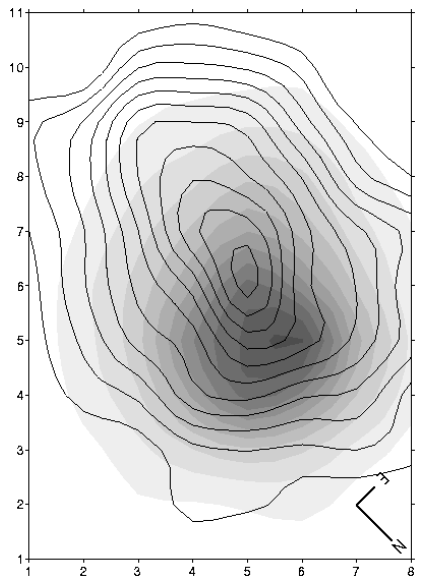

2.4 : Mrk 900

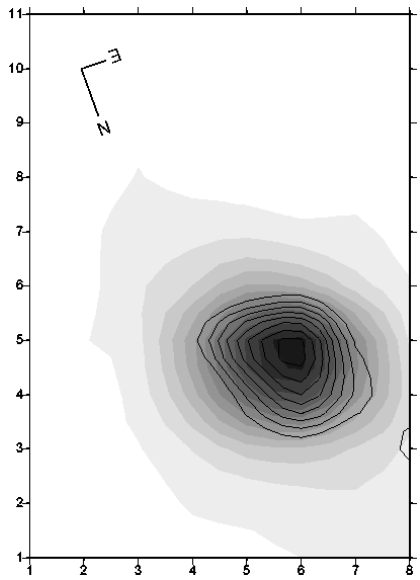

2.7 : Mrk 1434

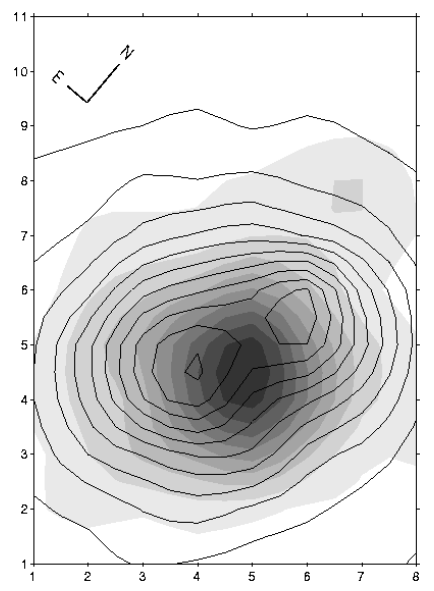

2.2 : Mrk 324

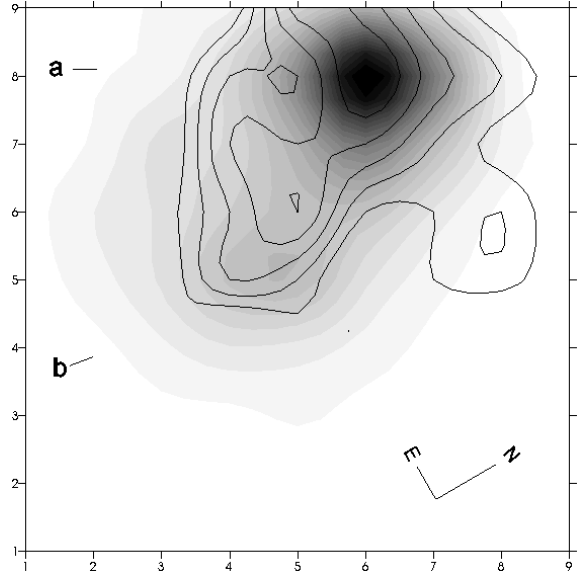

2.5 : Mrk 1416

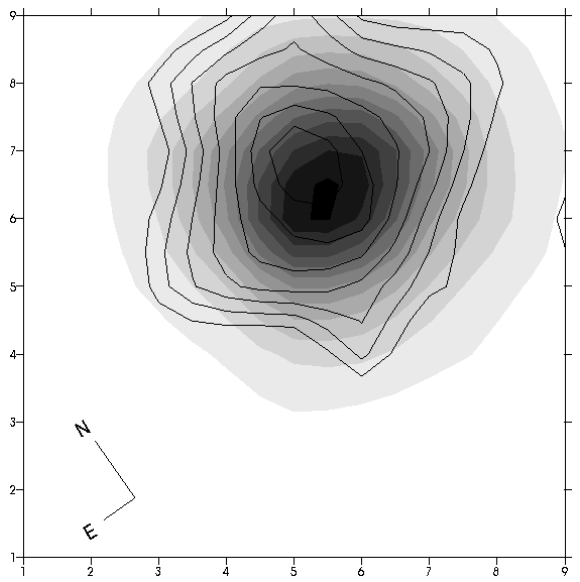

2.8 : Mrk 1450

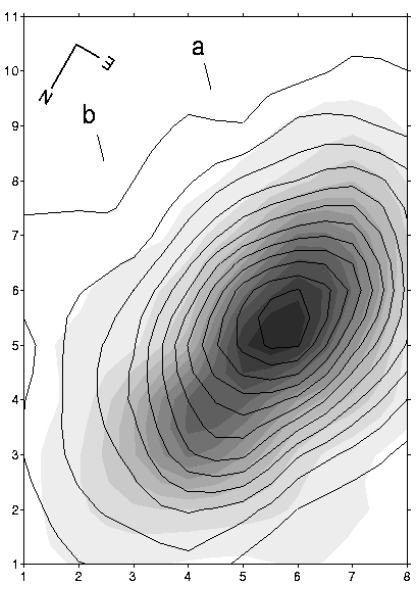

2.3 : Mrk 826

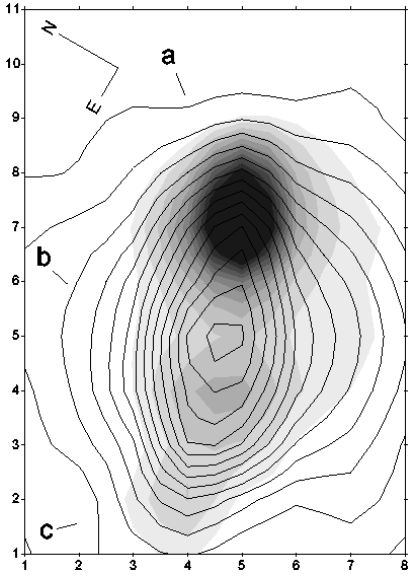

2.6 : Mrk 1426

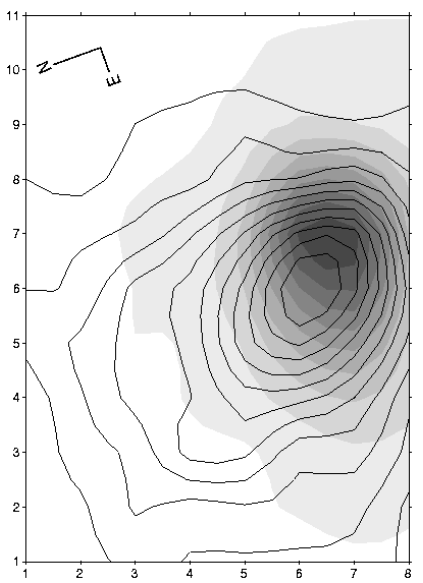

2.9 : Mrk 1499

Fig. 2. Distribution of $\mathrm{H} \alpha$ emission line intensity in grey halftone with the distribution of the red continuum integrated in a bandwidth of $400 \AA$ A overplotted as isophotal contours for 17 galaxies. The brightness scales are relative: i) isophotal step for H $\alpha$ : Mrk 1416, 1426: approx. $5 \%$ of maximum; SBS 1632+579, 1723+565B: approx. 15\% of maximum; ii) isophotal step for continuum: Mrk 900, 1416, 1426, 1450, SBS 1154+534, 1331+493, 1400+461, 1428+457: approx. 5\% of maximum; SBS 1723+565B: approx. 20\% of maximum. The tickmark interval on the frames sides is 1.3 arcsec. 


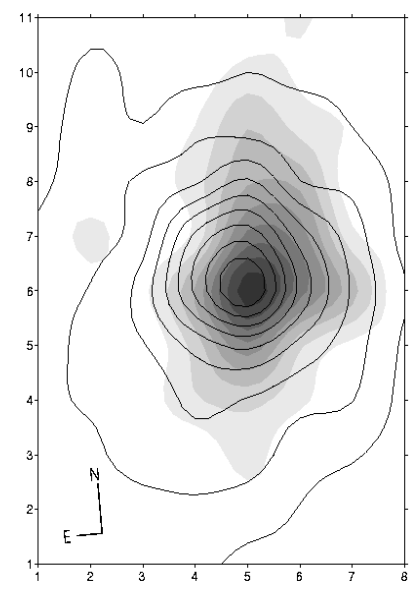

2.10 : SBS 0136+328

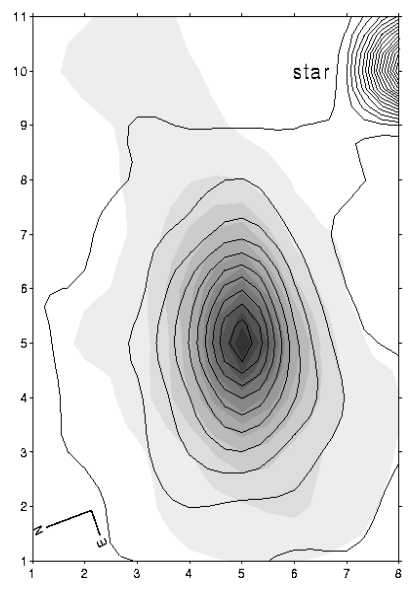

$2.13:$ SBS1400+461

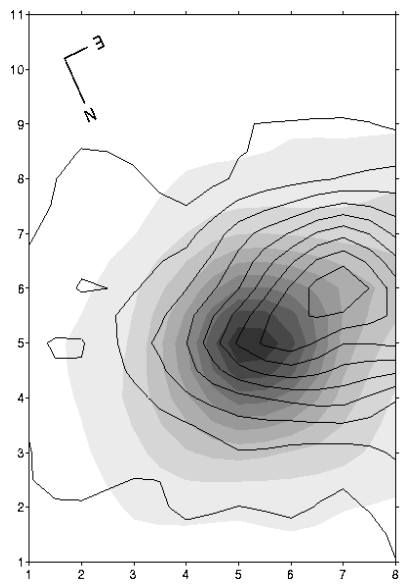

$2.16:$ SBS 1707+565

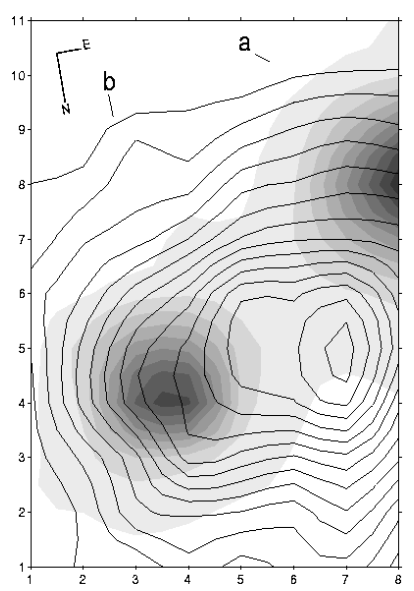

2.11 : SBS 1154+534

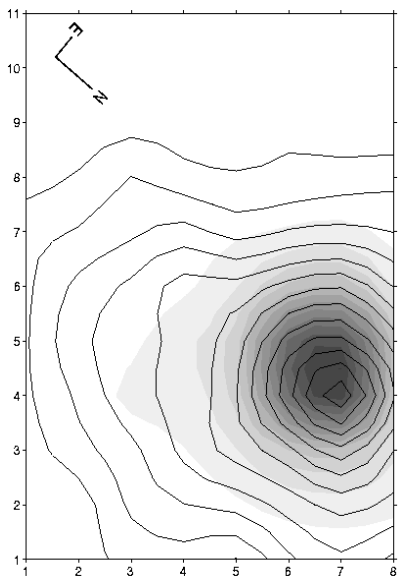

2.14 : SBS 1428+457

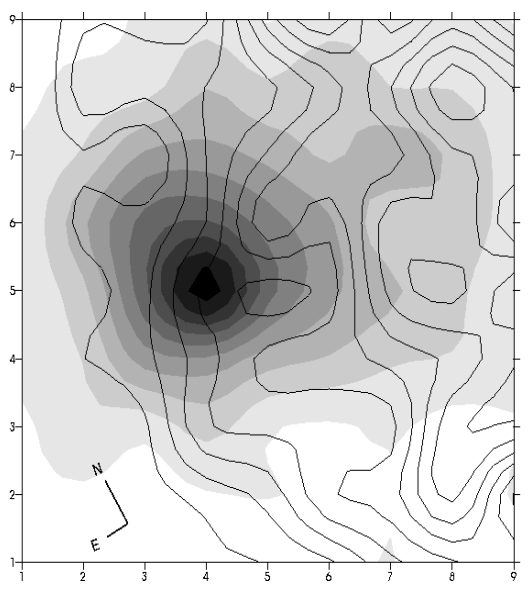

2.12 : SBS 1331+493

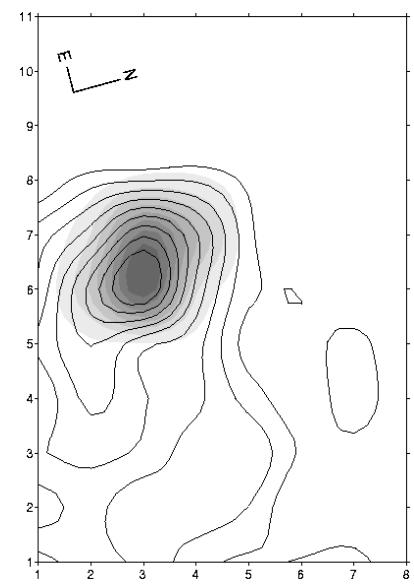

2.15 : SBS 1632+579

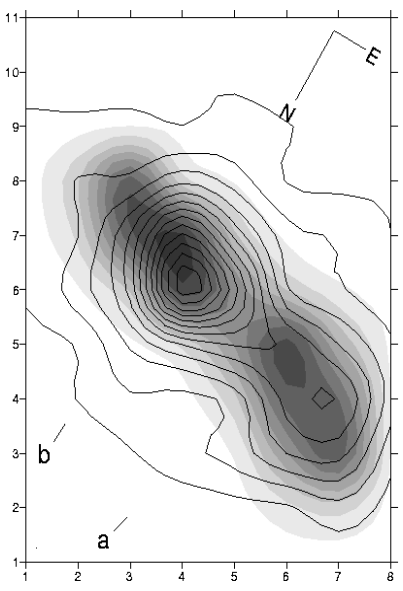

2.17 : SBS 1723+565

Fig. 2. continued. 


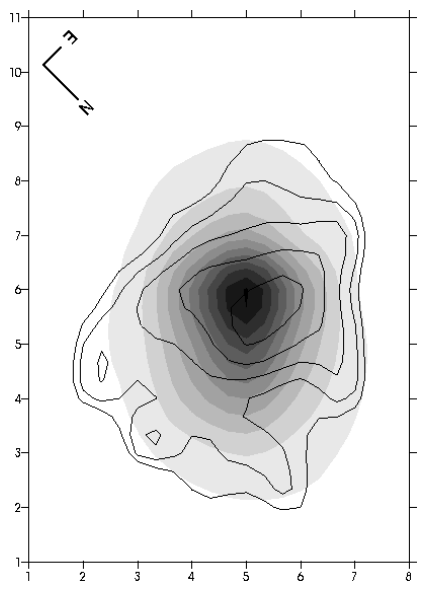

3.1 : Mrk 33

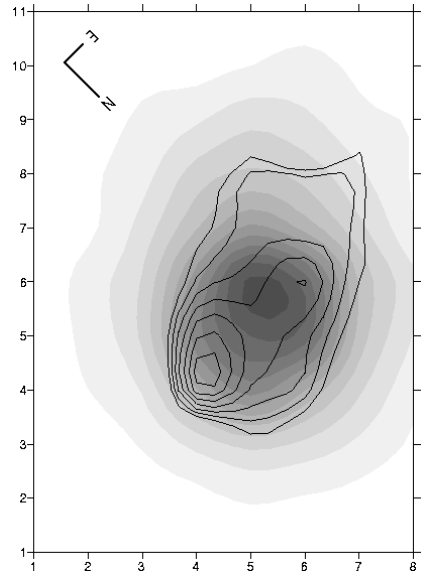

3.4 : Mrk 900

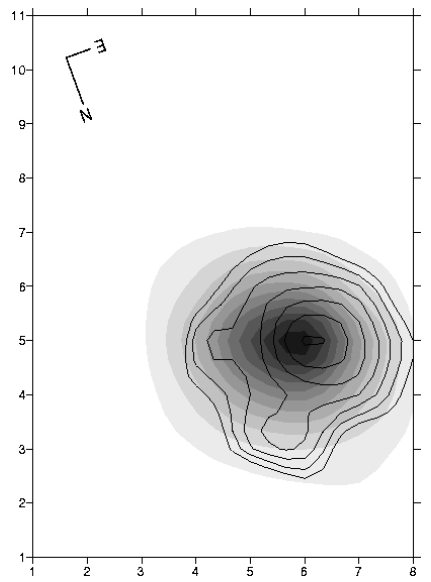

3.7 : Mrk 1434

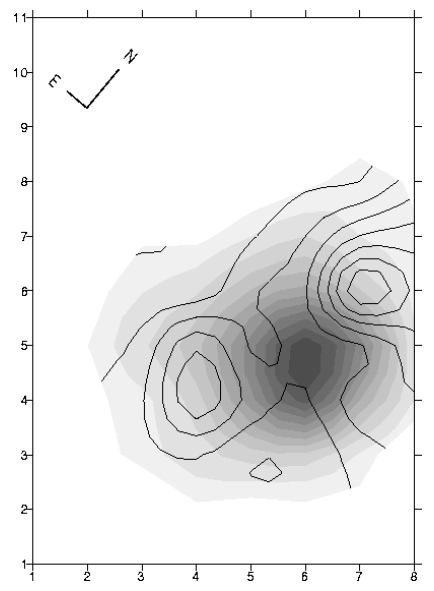

3.2 : Mrk 324

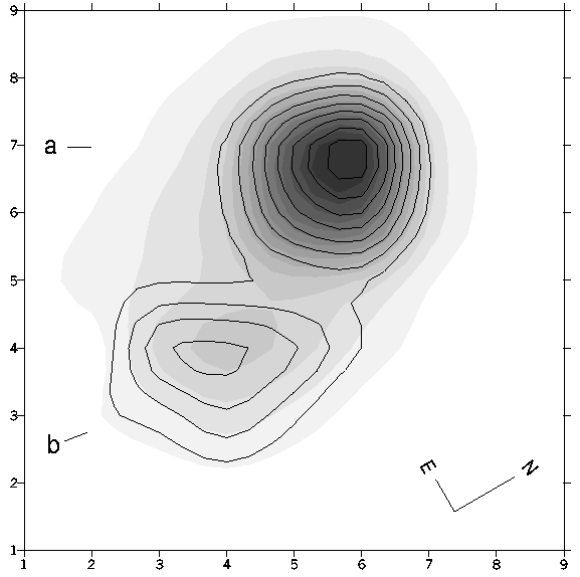

3.5 : Mrk 1416

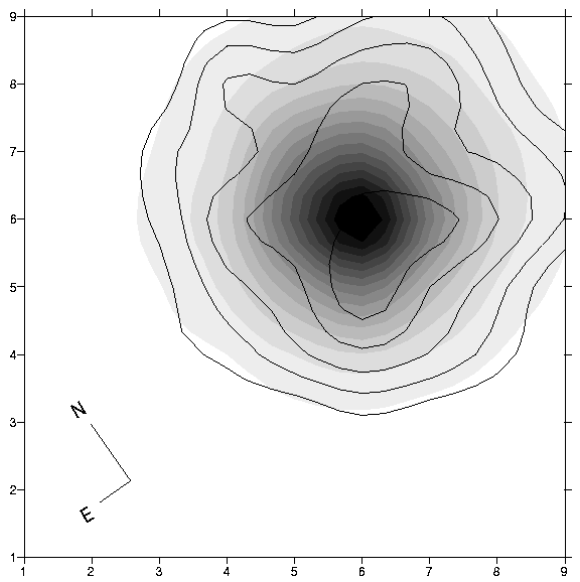

3.8 : Mrk 1450

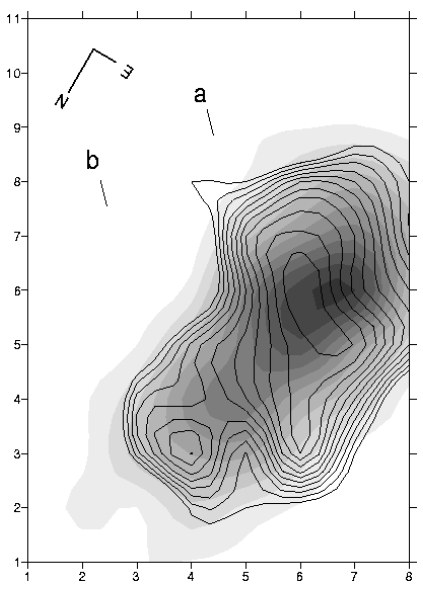

3.3 : Mrk 826

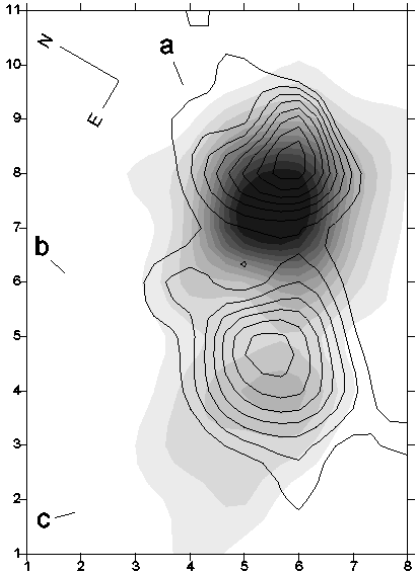

3.6 : Mrk 1426

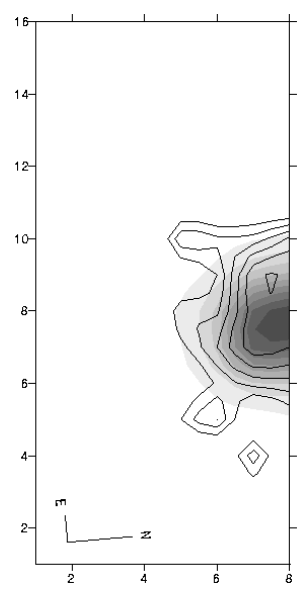

3.9 : Mrk 1480

Fig. 3. Distribution of the excitation ratio [OIII] $5007 \AA / \mathrm{H} \beta$ overplotted as isovalue contours on a grey half-tone map of the [OIII] $5007 \AA$. The isophotal step for [OIII] line is the same as in Fig. 1. For each object, the excitation isocontours are defined as follows (first number: external minimum plotted value, second number: step): Mrk 33 (2.5, 0.2); 324 (3.0, 0.4); 826 (3.0, 0.2); 900 (2.5, 0.2); 1416 (3.0, 0.5$) ; 1426(3.0,0.3)$; 1434 (4.5, 0.5); $1450(4.0,0.5) ; 1480(3.0,0.5) ; 1499(3.0,0.2)$; SBS 0136+328 (2.0, 0.2); 1154+534 (2.5, 0.5); $1331+493(4.0,0.5) ; 1400+461$ $(2.0,0.2) ; 1428+457(3.0,0.5) ; 1632+579(2.5,0.3) ; 1707+565(3.0,0.5) ; 1723+565 \mathrm{~B}(3.0,0.5)$. The tickmark interval is 1.3 arcsec. 


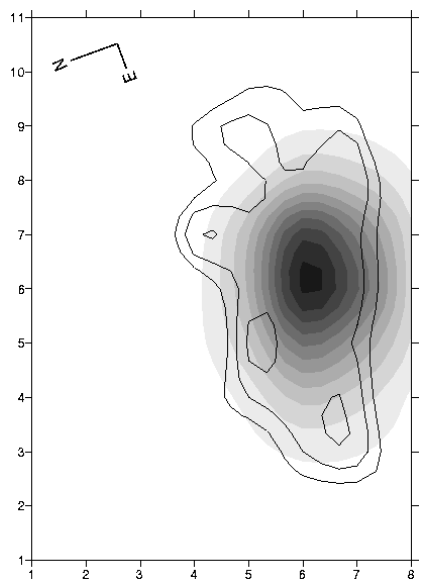

3.10 : Mrk 1499

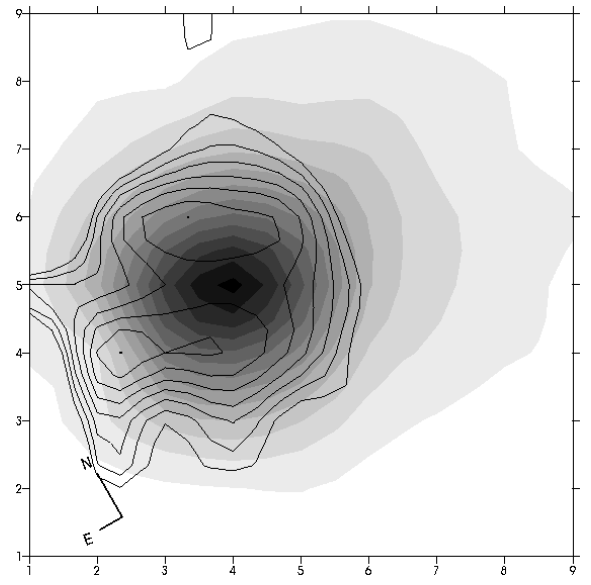

3.13 : SBS 1331+493

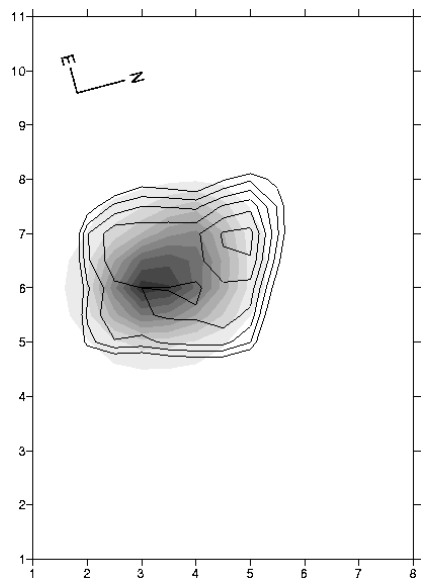

3.16 : SBS 1632+579

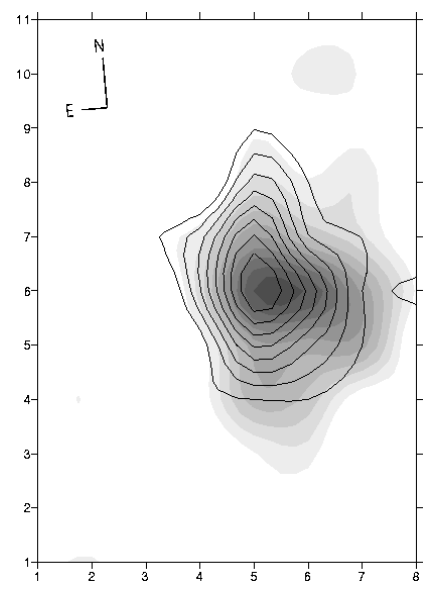

3.11 : SBS 0136+328

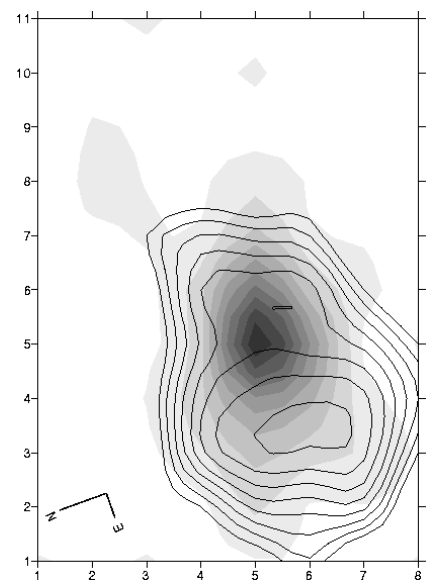

3.14 : SBS 1400+461

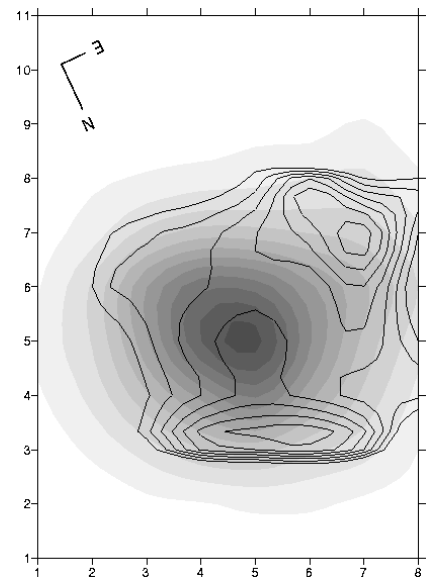

3.17 : SBS 1707+565

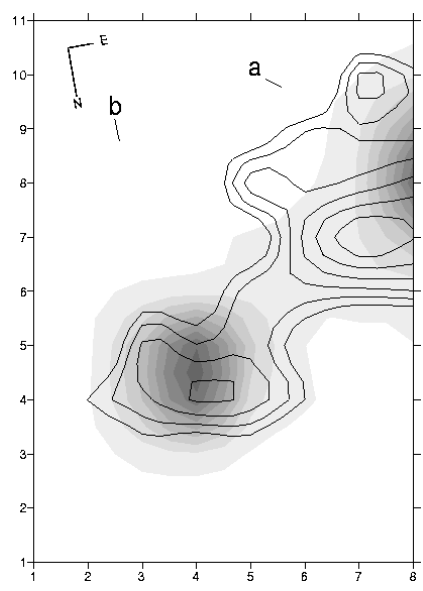

3.12 : SBS 1154+534

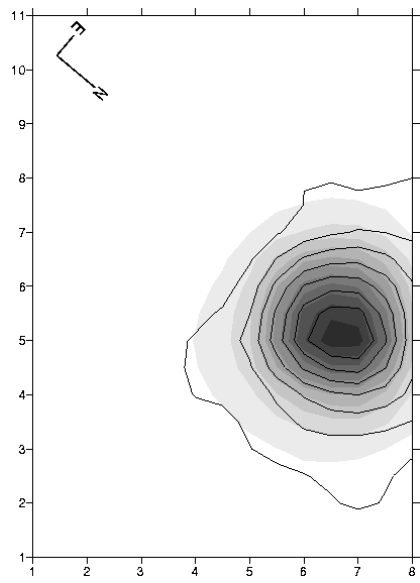

3.15 : SBS 1428+457

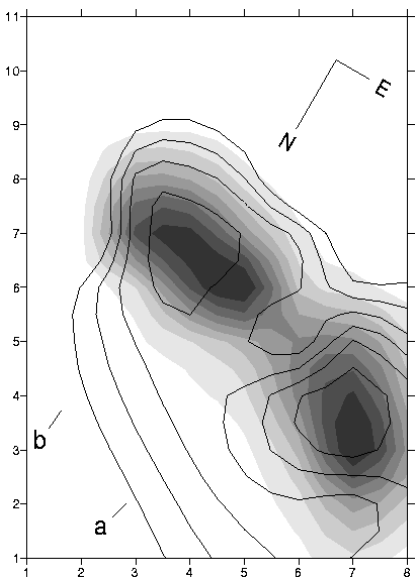

3.18 : SBS 1723+565

Fig. 3. continued. 


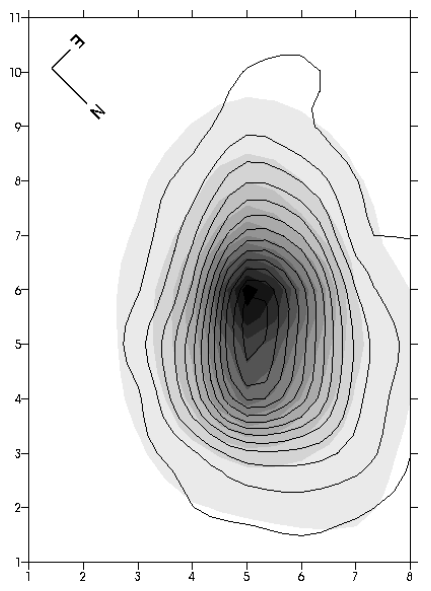

4.1 : Mrk 33

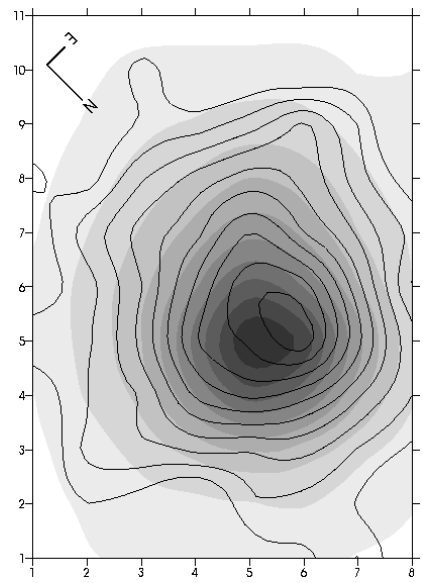

4.4 : Mrk 900

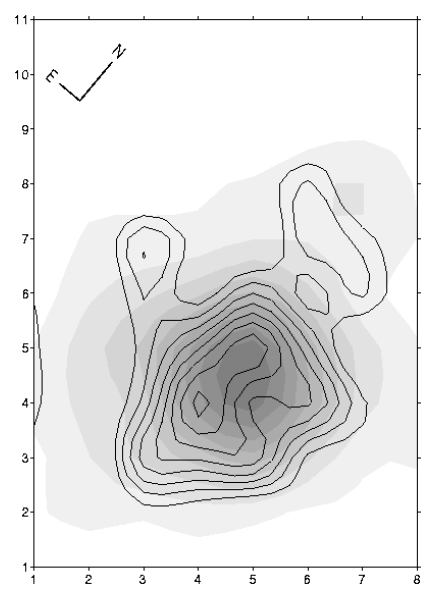

4.2 : Mrk 324

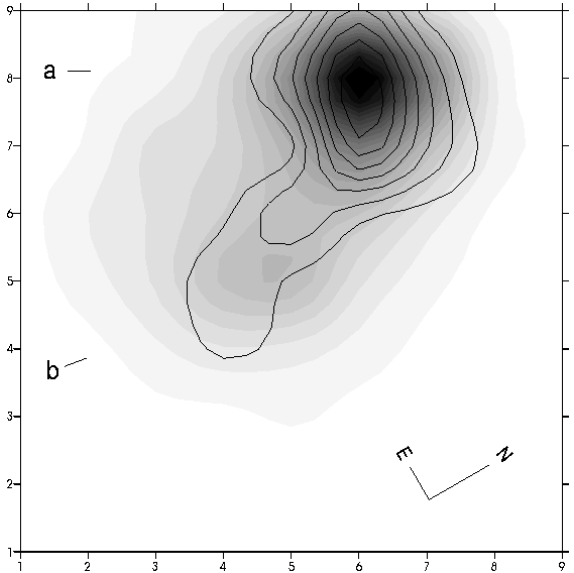

4.5 : Mrk 1416

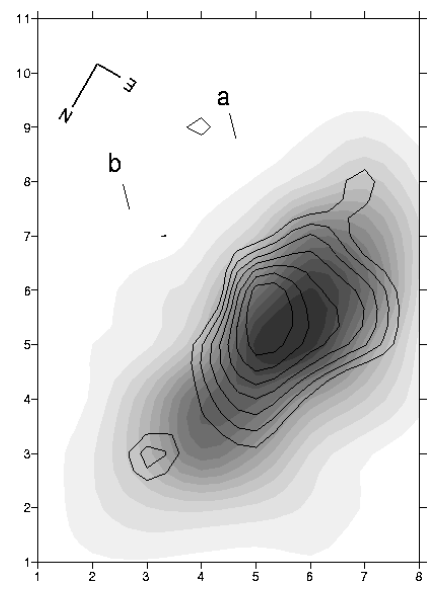

4.3 : Mrk 826

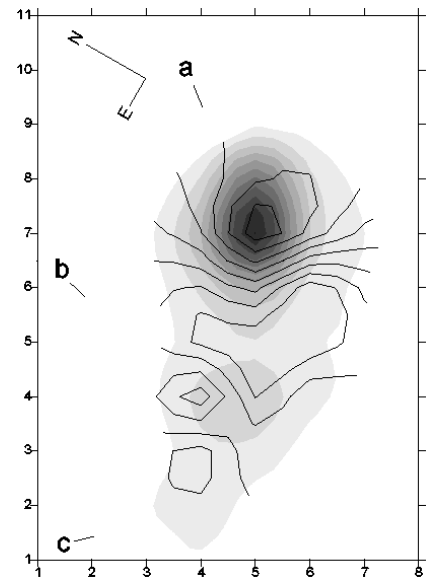

4.6 : Mrk 1426

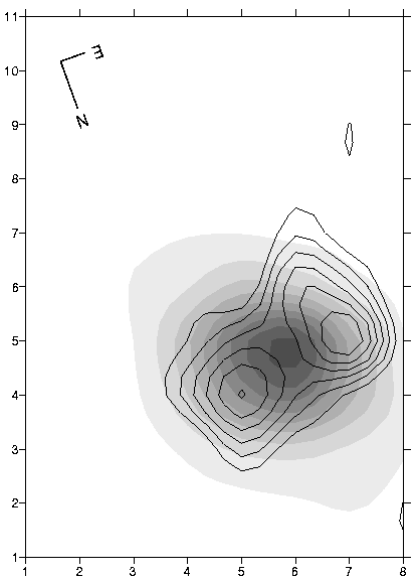

4.7 : Mrk 1434

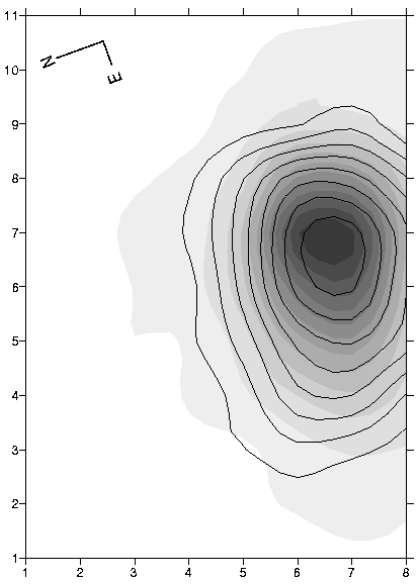

4.8 : Mrk 1499

Fig. 4. Distribution of [SII]6717+6731 А̊ emission line brightness overplotted as isophotal contours on a grey halftone map of H $\alpha$ emission for 13 galaxies. The brightness scales are relative: i) isophotal step for $\mathrm{H} \alpha$ is the same as on Fig. 2; ii) isophotal step for [SII]: Mrk 33, SBS 1154+534: approx. 5\% of maximum; SBS 1707+565: approx. 15\% of maximum. The tickmark interval on the frames sides is 1.3 arcsec. 


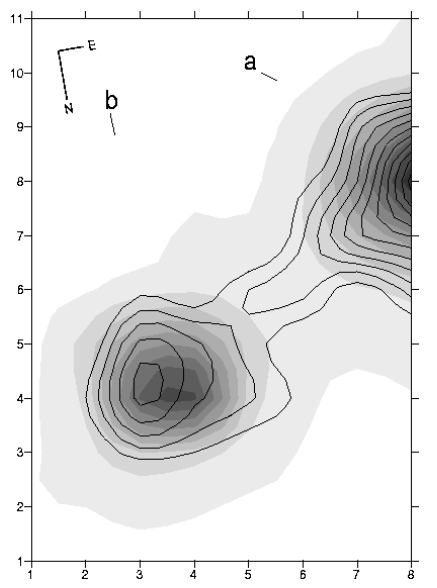

4.9 : SBS 1154+534

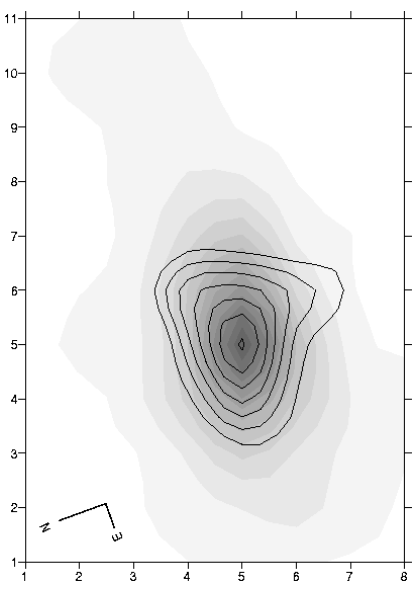

$4.10:$ SBS 1400+461

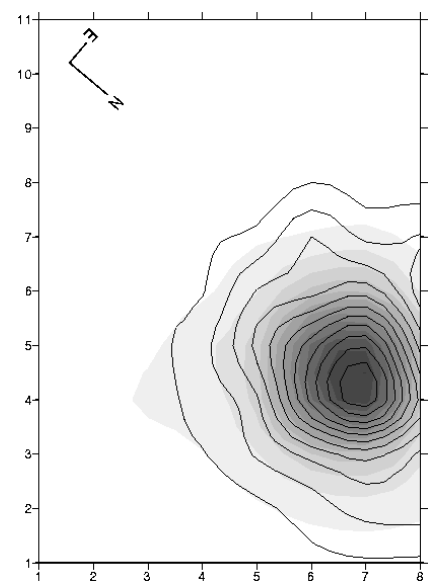

$4.11:$ SBS 1428+457

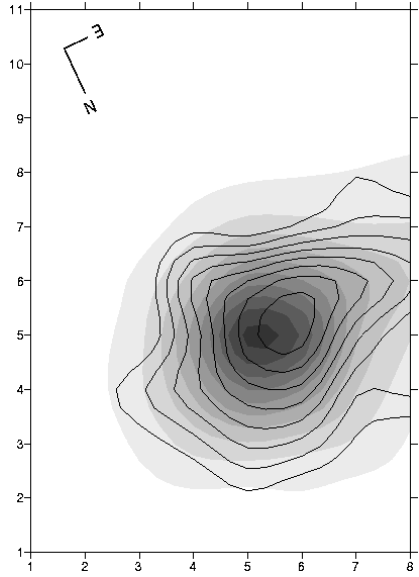

4.12 : SBS 1707+565

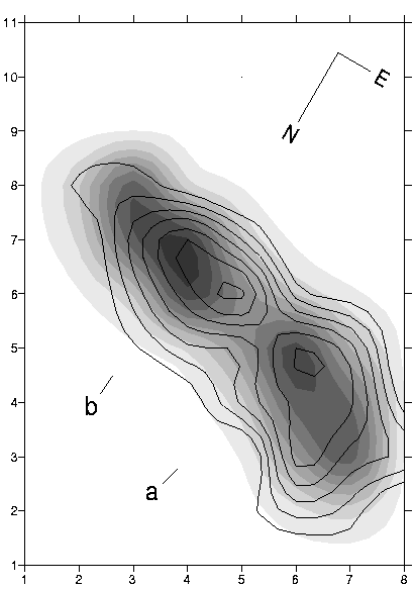

$4.13:$ SBS 1723+565

Fig. 4. continued.

This kinematical major axis was derived from the velocity field in order to obtain the maximum radial velocity gradient along it within a typical accuracy of 5 to 10 degree. Photometric major axises were measured with an accuracy better than 10 degree on the DSS-II blue images.

The average values of radial velocities given in Table 2 are in good agreement with published data (Thuan \& Martin 1981; Mazzarella \& Boroson 1993; Comte et al. 1999; Thuan et al. 1999).

\subsection{Comments on individual objects}

- Mrk 33 (Haro 2, Arp 233): Mrk 33 is the brightest known BCG $\left(m_{B}=13.4\right.$ and $M_{B}=-18.4$ from Loose \& Thuan (1986b) in our sample. Its brightness distribution is dominated by an $r^{1 / 4}$ law across a large part of the object.

Our observations cover the central $10 \times 14 \operatorname{arcsec}(1.0 \times$ $1.4 \mathrm{kpc}$ ) starburst component (Papaderos et al. 1996). Emission lines ratios and $E W(\mathrm{H} \beta)$ across this region (Table 2) are in good agreement with published ones obtained through larger apertures (Gallagher \& Hunter 1989; Kennicutt 1992). The continuum in blue and red is more extended than the emission lines but their peak intensities coincide in position (Figs. 1.1 and 2.1). In comparison with the continuum and emission lines intensity distributions, the $[\mathrm{OIII}] / \mathrm{H} \beta$ ratio map is more disturbed (Fig. 3.1).

The [SII] brightness map is similar to the continuum, [OIII] and Balmer lines ones (Fig. 4.1).

Mrk 33 shows a clear overall regular radial velocity gradient (Fig. 5.1), globally consistent with a solid-body-like rotation of the ionized gas. The kinematical major axis of this gas is oriented along the NS direction, differing by about 40 degree from the photometric major axis of the galaxy. The radial velocity gradient across the Southern part of the galaxy is much steeper $\left(0.260 \mathrm{~km} \mathrm{~s}^{-1} \mathrm{pc}^{-1}\right)$ than across its Northern part $\left(0.060 \mathrm{~km} \mathrm{~s}^{-1} \mathrm{pc}^{-1}\right)$. This picture compares well with the data obtained by Legrand et al. (1997) who obtained spectra through a slit positioned along the photometric major axis of the galaxy and also discovered a flatter velocity gradient. Assuming that the velocity gradient is due to rotation we 


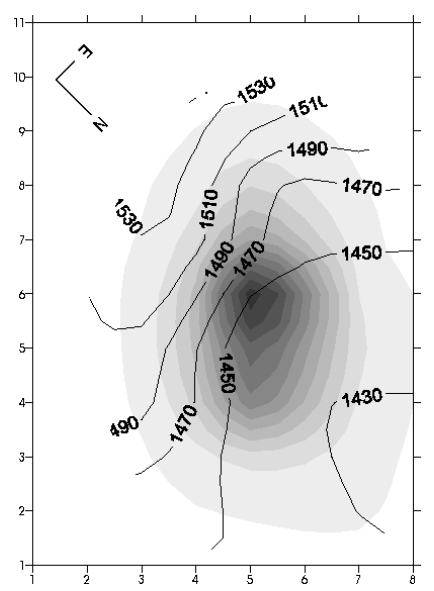

5.1 : Mrk 33

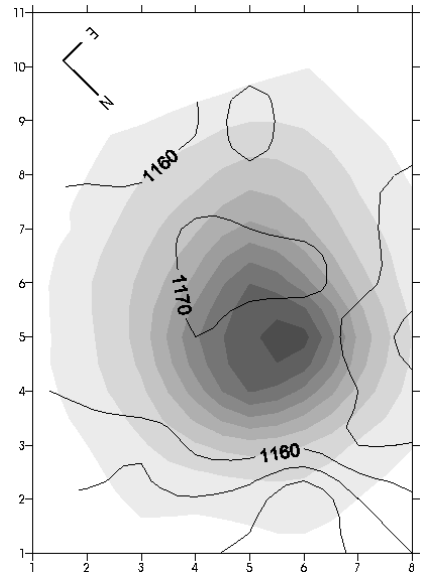

5.4 : Mrk 900

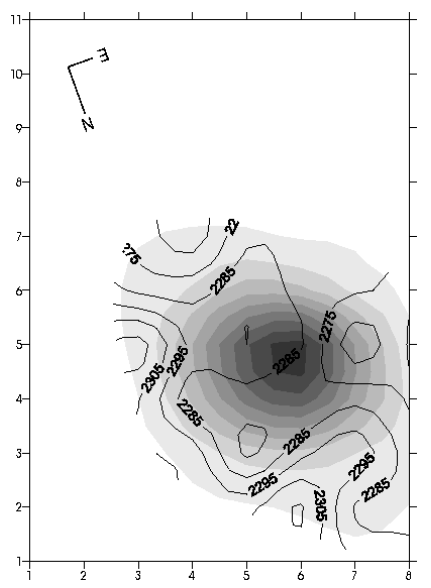

5.7 : Mrk 1434

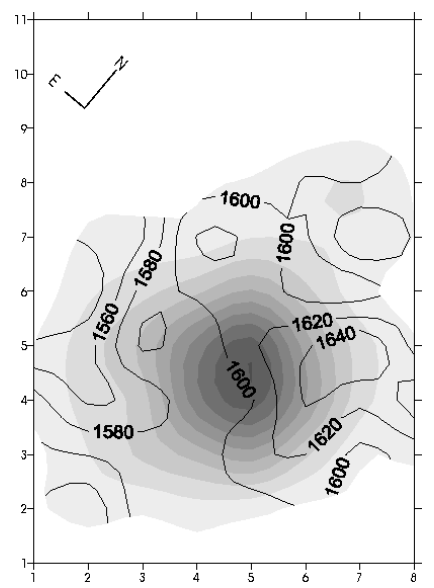

5.2 : Mrk 324

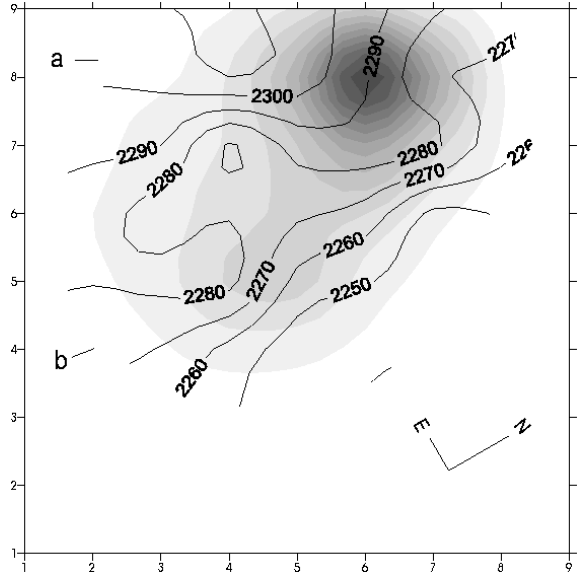

5.5 : Mrk 1416

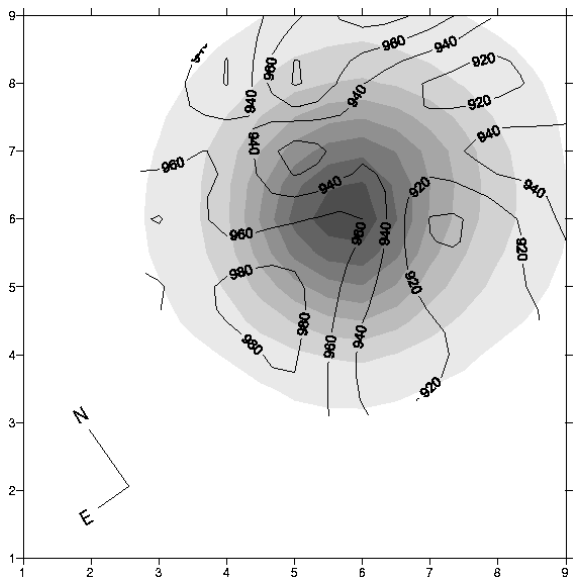

5.8 : Mrk 1450

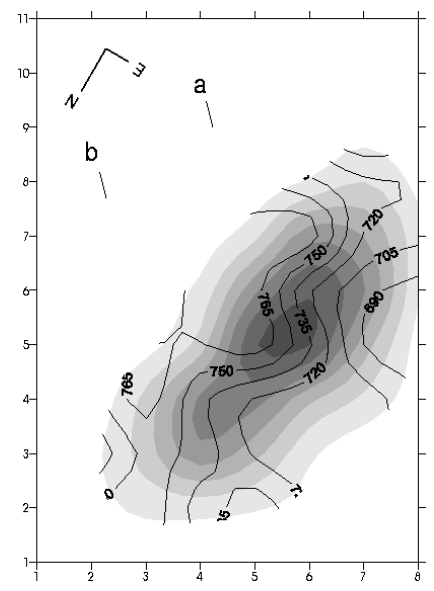

5.3 : Mrk 826

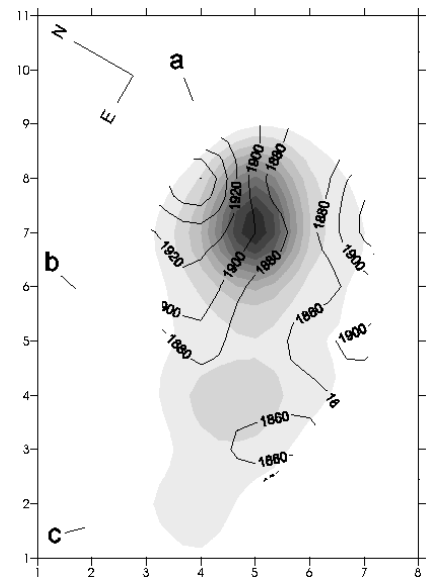

5.6 : Mrk 1426

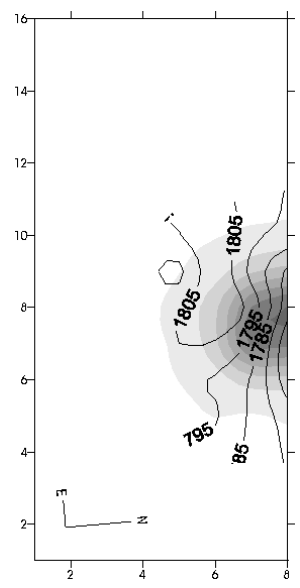

5.9 : Mrk 1480

Fig. 5. Velocity field of the 18 observed galaxies, derived from $\mathrm{H} \alpha$ emission line, except for Mrk 1480 for which the [OIII]5007 $\AA$ line was used. The isovelocities are overplotted on grey halftone maps of $\mathrm{H} \alpha$ brightness ([OIII] for Mrk 1480). The tickmark interval on the frames sides is 1.3 arcsec. 


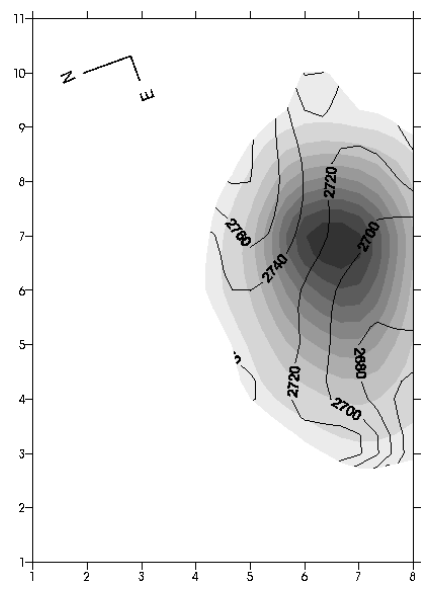

5.10 : Mrk 1499

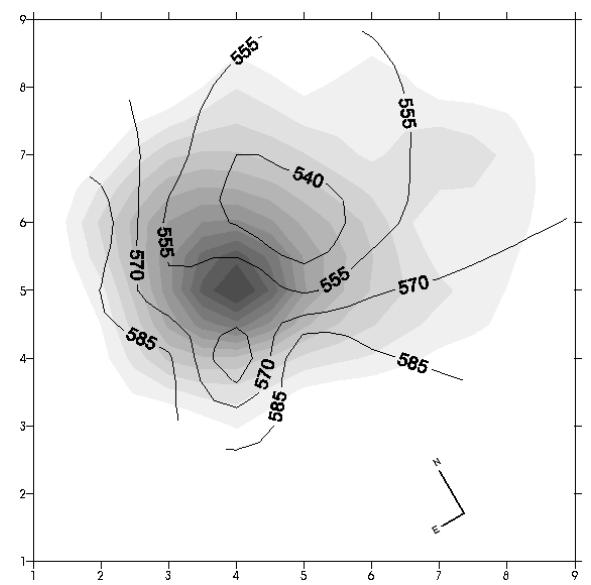

5.13 : SBS 1331+493

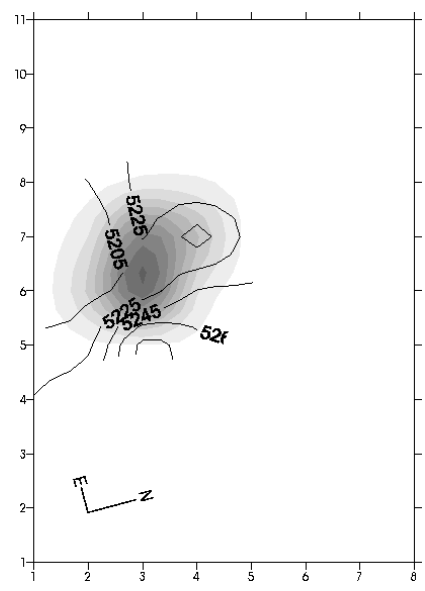

5.16 : SBS 1632+579

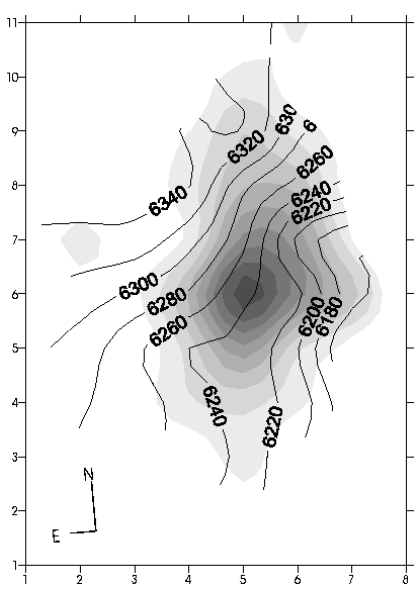

5.11 : SBS 0136+328

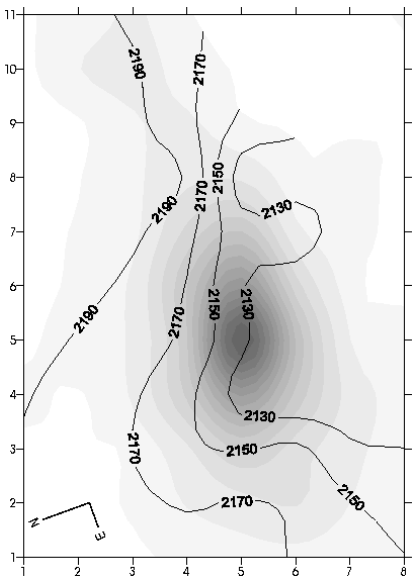

5.14 : SBS 1400+461

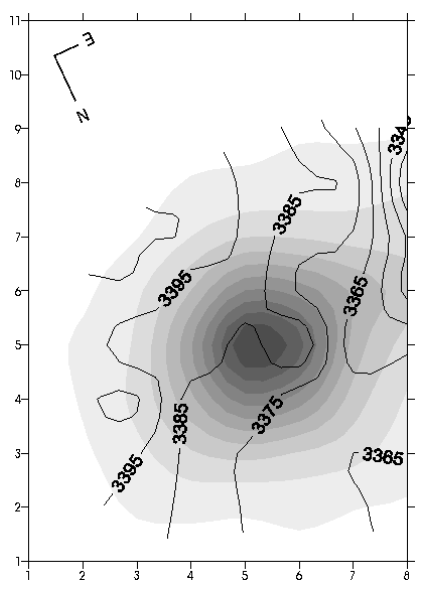

5.17 : SBS 1707+565

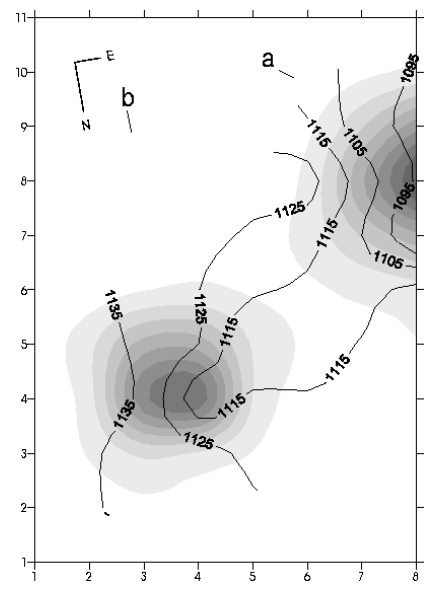

5.12 : SBS 1154+534

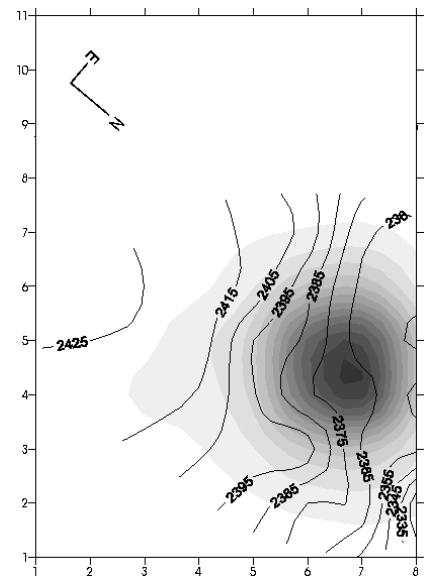

5.15 : SBS 1428+457

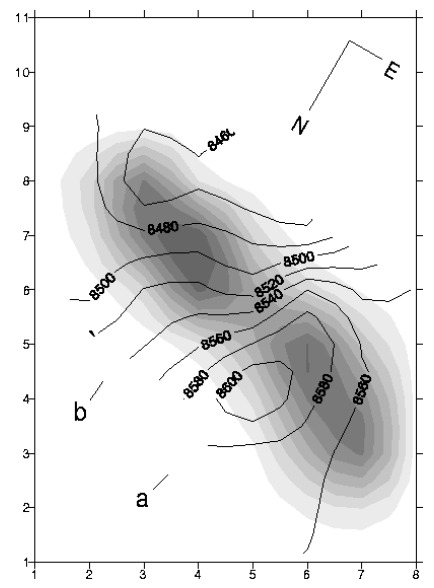

5.18 : SBS 1723+565

Fig. 5. continued. 
derive a Keplerian spherical mass of $4 \times 10^{8} M_{\odot}$ within a radius of 5 arcsec (about $500 \mathrm{pc}$ ), consistent with the spherical total mass determination of Loose \& Thuan (1986b).

- Mrk 324: this galaxy is a "double nucleus" object (Barbieri et al. 1979; Korovyakovskii et al. 1981; Mazzarella \& Boroson 1993; Doublier et al. 1997). The H $\alpha$ /red (Fig. 2.2) and [OIII]/blue continuum (Fig. 1.2) maps reveal a spectacular difference in the behaviour of the ionized gas with respect to the central stellar population. The line emission is widespread across the two central "nuclei" but its maximum brightness appears located between them. The $[\mathrm{OIII}] / \mathrm{H} \beta$ map (Fig. 3.2) shows a double-peaked distribution. Both excitation maxima lie on the line connecting the condensations as seen in continuum light and are located near their outer edges.

The [SII] map shows a brightness maximum splitted into two subcomponents clearly disconnected from the $\mathrm{H} \alpha$ peak (Fig. 4.2). The structure of the [SII] distribution could provide some evidence for the presence of a local, ringed shock encompassing an area of about 3 arcsec (about $300 \mathrm{pc}$ ) between the Southern stellar core and the position of the ionized gas maximum projected density.

The velocity field, although somewhat chaotic, still shows some underlying regular pattern. The equidistant isovelocities imply a solid-body like rotation across the central 10 arcsec (about $1000 \mathrm{pc}$ ) but with a line of nodes at 30 degrees from the axis joining the two stellar "nuclei" (Fig. 5.2). The velocity difference between the northern and the southern parts of the observed region amounts to $50 \mathrm{~km} \mathrm{~s}^{-1}$. Assuming solid body rotation we estimate a spherical mass of $5.7 \times 10^{7} M_{\odot}$ within a radius of 4 arcsec (about $400 \mathrm{pc}$ ).

- Mrk 826: this galaxy has a regular oval shape. Its apparent dimensions on the POSS-2 are $35 * 45$ arcsec. The $[\mathrm{OIII}] /$ blue and $\mathrm{H} \alpha$ / red continuum maps show that the emission lines spread across the whole central $13 * 8$ arcsec area (about $620 * 380 \mathrm{pc}$ ), in an elongated ridge forming a double ionized component (Figs. 1.3 and 2.3). The blue and red continuum maxima coincide quite well with the brightest, southern emission line component (labelled (a) on Figs. 1.3 and 2.3).

The excitation parameter [OIII]/H $\beta$ map gives a more complex pattern (Fig. 3.3). The strongest peak of this distribution roughly coincides with the position of the (a) component but exhibits an elongated extension along a NE-SW direction. A secondary maximum of the excitation parameter lies 1.7 arcsec (about 83 pc) $\mathrm{N}$ from the emission component (b).

In [SII] lines, the image of the galaxy (Fig. 4.3) seems limited to the (a) component.

The velocity field (Fig. 5.3) contains a clear regular pattern with slightly different gradients $\left(0.60 \mathrm{~km} \mathrm{~s}^{-1} \mathrm{pc}^{-1}\right.$ across the southern (a) condensation and $0.48 \mathrm{~km} \mathrm{~s}^{-1} \mathrm{pc}^{-1}$ across the northern one (b)). The kinematical line of nodes has a position inconsistent with the apparent morphological major axis of the galaxy (70 degrees difference). The isovelocity lines are equidistant but noticeably twisted. Assuming this gradient to be the signature of a solid body rotation we derive a total mass of $2 \times 10^{8} M_{\odot}$ within a radius of $3 \operatorname{arcsec}(140 \mathrm{pc})$.
- Mrk 900: Abrahamian \& Saakian (1989) refer to a "double nucleus" object, while Doublier et al. (1997) have found that this galaxy is dominated in its central part by a $r^{1 / 4}$ brightness distribution law, with boxy and rotating isophotes. The $\mathrm{H} \alpha /$ red continuum map (Fig. 2.4) exhibits a compact emission line region whose center is clearly displaced by 1.7 arcsec (about $130 \mathrm{pc}$ ) with respect to that of the slightly distorted continuum region. The same feature is visible on the [OIII] / blue continuum map, but the offset is somewhat reduced (Fig. 1.4).

The $[\mathrm{OIII}] / \mathrm{H} \beta$ ratio, mapped across a central $6.5 \times 5$ arcsec area, (Fig. 3.4) shows a double peaked structure: the secondary peak coincides with the maximum emission lines intensity but the primary one (highest values of the excitation parameter) is shifted at 2.3 arcsec to the west (about $170 \mathrm{pc}$ ).

The [SII] map (Fig. 4.4), approximately coincides with the distribution of $\mathrm{H} \alpha$ emission line.

The velocity field of the galaxy appears quite homogeneous (Fig. 5.4), clearly excluding any strong rotational gradient.

- Mrk 1416: according to Doublier et al. (1997), this comet-like galaxy has a double component structure and a brightness distribution consistent with the superimposition of two exponential laws. The emission-lines maps show the double component structure more clearly than in the continuum (Figs. 1.5 and 2.5).

$[\mathrm{OIII}] / \mathrm{H} \beta$ ratio and $[\mathrm{SII}]$ lines distributions mimic the double component structure seen in [OIII] and $\mathrm{H} \alpha$ (Figs. 3.5 and 4.5).

The emission lines ratios (Table 2) are in agreement with the data of Izotov et al. (1997). The difference in $E W(\mathrm{H} \beta)$ between our measurement (Table 2) and that of Izotov et al. (1997) (85 $\AA$ ) is large since our values are given for the whole body of the galaxy ( $3 \AA$ ) or (a) and (b) components ( $27 \AA$ and $10 \AA$ A respectively) while Izotov et al. (1997) use only a narrow slit across the central star-forming region.

The galaxy shows a regular velocity gradient (Fig. 5.5) which varies between $0.095-0.060 \mathrm{~km} \mathrm{~s}^{-1} \mathrm{pc}^{-1}$ across its main body. The kinematic major axis differs from the photometric major axis of the galaxy by 50 degrees. Assuming that the radial velocity gradient is due to solid body rotation we derive a total mass of $7.8 \times 10^{7} M_{\odot}$ within a radius of $2.5 \operatorname{arcsec}(370 \mathrm{pc})$.

- Mrk 1426: Doublier et al. (1997) describe an elliptically shaped galaxy with the $R$-band brightness distribution following an $r^{1 / 4}$ law. The central bright region exhibits a double component structure, the brightest one being centered with respect to outer isophotes. A multicomponent structure is detected in the central region of the galaxy in the emission lines ([OIII] and $\mathrm{H} \alpha$ ) but not in the continuum. The inclusion of the strong $\mathrm{H} \alpha$ emission-line in $R$-band photometry by Doublier et al. (1997) has probably led them to conclude to the presence of double stellar structure in this galaxy.

Figures 1.6 and 2.6 show three well separated emission lines components designated (a), (b), (c) respectively. The blue and red continuum isophotes, ellipticaly shaped, have a maximum brightness located between the (a) and (b) emission lines components. The $[\mathrm{OIII}] / \mathrm{H} \beta$ ratio distribution gives a double 
peaked structure (Fig. 3.6), but the peaks do not coincide with the position of the emission lines intensity maxima.

The [SII] lines distribution is smooth (Fig. 4.6) with a faint local maximum at the position of the (b) emission lines component.

The velocity field of the galaxy across the whole emission line region is nearly regular, (Fig. 5.6).

- Mrk 1434: this galaxy has a central bright component and a $r^{1 / 4}$ brightness distribution (Doublier et al. 1997). According to our data, the galaxy is regularly shaped in blue and red continuum as well as in the [OIII] and Balmer emission lines. The spatial coincidence of their maximum emission is excellent (Figs. 1.7 and 2.7). The excitation map provides approximately the same shape, (Fig. 3.7).

The [SII] lines map shows a double peaked structure (Fig. 4.7): its maxima lie symmetrically on western and eastern sides of the $\mathrm{H} \alpha$ peak.

The emission lines ratios in Table 2 are in agreement with the data of Izotov et al. (1997).

The velocity field across the central region of the galaxy appears globally homogeneous (Fig. 5.7) around an average velocity of $2280 \mathrm{~km} \mathrm{~s}^{-1}$. But the local velocity maxima of the eastern (about $2275 \mathrm{~km} \mathrm{~s}^{-1}$ ) and north-western (about $2270 \mathrm{~km} \mathrm{~s}^{-1}$ ) regions of the galaxy coincide well with the [SII] lines maxima (Fig. 4.7). It is likely that we observe a superbubble structure originating in violent star formation and subsequent superwind driven from a single stellar supercluster.

- Mrk 1450: very compact object (it has been misclassifed as a star by Munari et al. 1995) whose brightness distribution follows an $r^{1 / 4}$ law with a single central star forming component (Doublier et al. 1997). These properties are well seen in Figs. 1.8 and 2.8. The $[\mathrm{OIII}] / \mathrm{H} \beta$ ratio map (Fig. 3.8) shows a distribution peak slightly displaced southwards from the intensity maximum of the emission lines and the continuum. Our integral emission line ratios (Table 2) agree with Izotov et al. (1997).

The velocity field is roughly homogeneous (Fig. 5.8) with a local maximum at 2.7 arcsec from the center. There is no obvious regular gradient that could identify any ordered rotation.

- Mrk 1480: a well shaped galaxy with a $r^{1 / 4}$ law brightness distribution and a single bright knot in the center (Doublier et al. 1997). Our observations are limited to the blue spectral region and suffer from a pointing problem that projected the galaxy onto the edge of the microlens array.

The [OIII] over blue continuum map shows that this line emitting region is more compact and highly concentrated with respect to the stellar component (Fig. 1.9). The $[\mathrm{OIII}] / \mathrm{H} \beta$ excitation parameter map coincides approximately with the [OIII] line intensity distribution (Fig. 3.9).

The velocity field of the galaxy, built from the [OIII] line limited to the southern part of the central knot (Fig. 5.9) is regular with a velocity difference between the center and the edge of about $30 \mathrm{~km} \mathrm{~s}^{-1}$. If real, and symmetrical across the northern half of the knot, this difference may be due to ordered rotation. The limited amount of the present data however prevents a firm conclusion.
- Mrk 1499 (I Zw 159): Doublier et al. (1997) describe an irregular galaxy with a very blue (see also Moles et al. 1987) elongated central structure with two components. Our continuum maps reproduce the elongation of the central structure along the same PA as observed by Doublier et al. (1997). Emission lines (Figs. 1.10 and 2.9) have a brightness distribution with a different major axis, 35 degree from that of the continuum. We do not confirm the presence of a double component structure in the galaxy core, neither in continuum nor in emission lines. This elusive structure claimed by Doublier et al. (1997) may be an observational artefact. The $[\mathrm{OIII}] / \mathrm{H} \beta$ ratio map reveals a spatially rather homogeneous excitation across the line emitting region (Fig. 3.10).

The distribution of [SII] lines emissivity coincides especially well with the $\mathrm{H} \alpha$ emission line intensity distribution (Fig. 4.8).

The central region of the galaxy shows an overall regular velocity gradient (Fig. 5.10) of $0.115 \mathrm{~km} \mathrm{~s}^{-1} \mathrm{pc}^{-1}$ as measured along the line of nodes. This kinematical major axis differs by some 60 degree from the position angle of the photometric major axis of the galaxy. Assuming that the radial velocity gradient is due to solid body rotation, we derive a total (spherical) mass of $3.6 \times 10^{8} M_{\odot}$ within a radius of $2.8 \operatorname{arcsec}(490 \mathrm{pc})$.

- SBS 0136+328: quite luminous and distant oval galaxy with a large, dominant $r^{1 / 4}$ bulge embedded in an exponential envelope structure (Doublier et al. 1997). The brightness distributions in blue and red are roughly elliptically shaped and their peaks coincide with those of the emission lines (Figs. 1.11 and $2.10)$, the latter being irregular in shape and less extended. The $[\mathrm{OIII}] / \mathrm{H} \beta$ distribution is fairly similar to the [OIII] emission line intensity distribution (Fig. 3.11).

This galaxy exhibits a clear overall regular radial velocity field (Fig. 5.11), consistent with a solid-body-like rotation. The kinematic major axis differs by about 33 degree from the adopted photometric major axis of the galaxy. The radial velocity gradient is $0.080 \mathrm{~km} \mathrm{~s}^{-1} \mathrm{pc}^{-1}$ which leads to a spherical mass of $1.7 \times 10^{9} M_{\odot}$ within a radius of $2.6 \operatorname{arcsec}$ (about $1050 \mathrm{pc}$ ).

- SBS 1154+534 (UGC 06923): known as a dwarf irregular satellite of the giant spiral galaxy NGC3992 (e.g. Erickson et al. 1999).

On both the red and blue contimuum images the central region of this system shows a bright core with an asymmetric brightness distribution. (Figs. 1.12 and 2.11). We suspect the existence of a "double nucleus" structure in the central stellar population. The emission lines of $\mathrm{H} \alpha$ and [OIII]5007 have a strikingly different distribution from that of the continuum, with two well separated components. The distance between them is approximately 7.8 arcsec (about $560 \mathrm{pc}$ ).

The $[\mathrm{OIII}] / \mathrm{H} \beta$ ratio distribution (Fig. 3.12) shows a complex pattern, with a peaked elongated $(E W)$ structure in the position of the (b) emission lines component while at the position of the (a) component, the peak of excitation lies 2 arcsec (about $140 \mathrm{pc}$ ) NW from its center. A secondary peak is observed $2.6 \operatorname{arcsec}(185 \mathrm{pc}) \mathrm{SW}$ from the (a) component. 
The [SII] lines distribution coincides remarkably well with the $\mathrm{H} \alpha$ distribution (Fig. 4.9). On the "total" spectrum derived from MPS data, an underlying $\mathrm{H} \beta$ absorption line is clearly visible, implying the presence of a quite rich A-F main sequence population.

The HI velocity field of the galaxy along its apparent elongation axis shows a weak velocity gradient of the order of $0.009 \mathrm{~km} \mathrm{~s}^{-1} \mathrm{pc}^{-1}$ (Sanders et al. 1998). No such velocity gradient could be seen across the MPS studied field (Fig. 5.12) We measured a velocity difference of $22 \pm 18 \mathrm{~km} \mathrm{~s}^{-1}$ between (a) and (b). This may suggest no special kinematical behaviour across the central region of the object with respect to the large scale motions of the galaxy.

- SBS 1331+493: this galaxy has an amorphous shape, a very complex central structure and a brightness distribution that does not obey to any standard laws (Doublier et al. 1997). Izotov et al. (1997) and Thuan et al. (1995) have published detailed spectrophotometric investigations, respectively of the North and South condensations.

Our MPS observations cover the East knot in the central part of the galaxy: we find (Table 2) similar excitation properties as in the North condensation (Izotov et al. 1997) while the $\mathrm{H} \beta$ line equivalent width is similar to that observed in the South condensation (Thuan et al. 1995). The E knot is a compact and well individualized object seen only in emission lines (Figs. 1.13 and 2.12) while a faint blue continuum (Fig. 1.13) probably traces a ionizing stellar cluster undetected in red (Fig. 2.12).

The distribution of the $[\mathrm{OIII}] / \mathrm{H} \beta$ ratio across the $\mathrm{E}$ knot shows a complex structure (Fig. 3.13), where the excitation maxima do not coincide with the location of the [OIII] and $\mathrm{H} \alpha$ peaks.

The observed knot shows a regular velocity gradient of $0.250 \mathrm{~km} \mathrm{~s}^{-1} \mathrm{pc}^{-1}$ along the WE direction (Fig. 5.13) with local perturbations of the isovelocities.

- SBS 1400+461: in continuum light as well as in emission lines this galaxy shows an elongated structure, with one single maximum of brightness at the same location (Figs. 1.14 and 2.13).

The $[\mathrm{OIII}] / \mathrm{H} \beta$ ratio (Fig. 3.14) shows a double peak distribution with a secondary maximum nearly coincident with the continuum and emission lines brightness peaks but a primary peak of excitation ratio lies 2.3 arcsec (about 325pc) $\mathrm{N}$ from the brightness maximum .

The [SII] lines intensity map, to a smaller extent, reproduces the morphology observed in continuum light, [OIII] and $\mathrm{H} \alpha$ lines (Fig. 4.10).

The velocity field has a regular pattern of solid-body like rotation (Fig. 5.14) with a line of nodes, differing by about 70 degrees from the apparent photometric major axis of the galaxy. The radial velocity gradient across the kinematic major axis is $0.10 \mathrm{~km} \mathrm{~s}^{-1} \mathrm{pc}^{-1}$. Assuming circular rotation we derive a spherical mass of $2.6 \times 10^{8} M_{\odot}$ within a radius of $3.5 \operatorname{arcsec}$ (about $480 \mathrm{pc}$ ).
- SBS 1428+457: Doublier at al. (1997) describe an object with a very high surface brightness central region and whose brightness ditribution is dominated by an exponential law.

The central star-forming region of this galaxy has been only partially covered by our observations. Both $\mathrm{H} \alpha$ over red continuum and [OIII] over blue continuum maps give the picture of an unresolved star-forming core of about 5 arcsec (about $765 \mathrm{pc}$ ) size with a fairly good correspondance between the continuum and the emission lines distributions (Figs. 1.15 and 2.14).

Distributions of the $[\mathrm{OIII}] / \mathrm{H} \beta$ ratio (Fig. 3.15) as well as [SII] (Fig. 4.11) show the same structure as in the continuum and line emission.

The velocity field shows a very regular pattern of solidbody like rotation (Fig. 5.15) with a kinematical major axis in PA 150 degrees, consistent with the apparent major axis of the optical image of the galaxy, with a radial velocity gradient along this axis equal to $0.070 \mathrm{~km} \mathrm{~s}^{-1} \mathrm{pc}^{-1}$. Assuming circular rotation, we derive a spherical mass of $3.7 \times 10^{8} M_{\odot}$ within a radius of $4.5 \operatorname{arcsec}$ (about $690 \mathrm{pc}$ ).

-SBS 1632+579: [OIII] and $\mathrm{H} \alpha$ emission lines maps show a compact distribution (Figs. 1.16 and 2.15) while the blue and red continuum are much more extended. Maxima observed in continuum and emission lines coincide spatially well with each other.

At the position of the emission lines and continuum peaks the $[\mathrm{OIII}] / \mathrm{H} \beta$ ratio shows a local minimum (Fig. 3.16). The excitation map shows a shallow concentration and its maximum is shifted by 2.4 arcsec (about $805 \mathrm{pc}$ ) $\mathrm{N}$ from the emission lines intensity maximum.

The velocity field (Fig. 5.16) is smooth with a local jump of velocity in its western part, whose reality however remains uncertain.

- SBS 1707+575: the elongated structure of this object is apparent on both blue and red continuum maps (Figs. 1.17 and 2.16), but is less noticeable in $\mathrm{H} \alpha$ (Fig. 2.16) and disappears in [OIII] line where the structure is more circular (Fig. 1.17). The central peak of continuum radiation does not coincide with emission lines maxima.

The $[\mathrm{OIII}] / \mathrm{H} \beta$ ratio distribution shows a disturbed structure (Fig. 3.17). In the central $3 \operatorname{arcsec}(650 \mathrm{pc})$ region of the [OIII] emitting knot, the excitation ratio is quite homogeneous. Two strong elongated peaks of the excitation parameter are located respectively at 3.7 arcsec or about $800 \mathrm{pc} E$ and 2.7 arcsec or $590 \mathrm{pc} \mathrm{N}$ from the emission line peak.

The [SII] lines intensity distribution shows a single component whose peak is only slightly offset from that of the $\mathrm{H} \alpha$ intensity distribution (Fig. 4.12); a "ridge" of [SII] brightness extends in the E direction across 2-3 arcsec (400-600 pc) following the elongation of the $\mathrm{H} \alpha$ structure.

The galaxy shows a regular radial velocity gradient (Fig. 5.17) with local perturbations of the isovelocities, with a line of nodes in PA 100 degrees, in fair agreement with the photometric major axis of the galaxy. Our recession velocity (Table 2) agrees with that given by Pustilnik et al. (1995). The radial velocity gradient across the kinematic major axis of the galaxy is $0.027 \mathrm{~km} \mathrm{~s}^{-1} \mathrm{pc}^{-1}$ which gives, assuming circular 
rotation, a spherical mass of $1.6 \times 10^{8} M_{\odot}$ within a radius of $4.5 \operatorname{arcsec}(980 \mathrm{pc})$.

- SBS 1723+565B: this object is in angular vicinity to the dwarf galaxy SBS $1723+565 \mathrm{~A}$ whose heliocentric velocity is $3149 \mathrm{~km} \mathrm{~s}^{-1}$ and absolute magnitude is -16.1 (Comte et al. 1999), but the large velocity difference excludes any physical neighbourhood.

This galaxy has not yet been studied. We suspected it to be a dwarf candidate because of its vicinity to SBS $1723+565 \mathrm{~A}$. In fact it proved the most distant galaxy of the present sample with a redshift of 0.02839 and an absolute magnitude of -19.7 (from an estimated blue apparent magnitude of 15.5).

Our continuum maps clearly show a double structure. The component (b) is roughly circular in shape and much brighter than the other one, especially in red. The (a) component is elongated (Figs. 1.18 and 2.17).

In both [OIII]5007 and $\mathrm{H} \alpha$ (Figs. 1.18 and 2.17) there appear two HII complexes that grossly correspond to the (a) and (b) continuum components but the emission line distributions are very elongated. In the (b) component the $\mathrm{H} \alpha$ and [OIII] maxima nearly coincide with the continuum peak. Note that a large elongation of the line emitting region is probably a secondary knot with a strong [OIII] emission at about $1.7 \operatorname{arcsec}(940 \mathrm{pc}) \mathrm{W}$ from the main knot. In the (a) component, the $\mathrm{H} \alpha$ coincides with the continuum peak but not the [OIII] maximum, clearly displaced towards the East.

The $[\mathrm{OIII}] / \mathrm{H} \beta$ map reflects the distribution in $[\mathrm{OIII}]$ and $\mathrm{H} \alpha$ lines (Fig. 3.18).

On the [SII] map (Fig. 4.13) two peculiar features appear: a maximum in [SII], on the South-East edge of the (b) component and a North-East extension from the main (a) component that does not follow the $\mathrm{H} \alpha$ distribution. These areas may contain shocked gas.

The galaxy shows a clear overall regular radial velocity gradient (Fig. 5.18) with a kinematical major axis differing by about 30 degree from the photometric major axis. The radial velocity gradient across the kinematic major axis is $0.065 \mathrm{~km} \mathrm{~s}^{-1} \mathrm{pc}^{-1}$. Assuming circular rotation we derive a spherical mass of $1.5 \times 10^{10} M_{\odot}$ within a radius of 4.5 arcsec (about $2.5 \mathrm{kpc}$ ), confirming the giant character of the object.

\section{Discussion}

This paper presents the most extensive study made to date of the 2-D structure of 17 BCDGs and the SBS $1723+565 \mathrm{~B}$ galaxy. This has been performed by using the blue and the red integrated stellar continuum, the [OIII] 5007, $\mathrm{H} \alpha$, and [SII] emission lines and the mapping of the $[\mathrm{OIII}] / \mathrm{H} \beta$ excitation ratio. Optical velocity fields of the ionized gas component have been built from the $\mathrm{H} \alpha$ or [OIII]5007 lines so as to cover the central regions of the objects. All observations have been made using the same equipment.

If we assume that stellar populations of different ages are responsible of the observed structures and of the spatial variations of the excitation parameter, we may interpret these observational facts in terms of SF history. However, this interpretation would be valid only under the hypothesis that the Initial
Mass Function (IMF) is nearly the same in all objects (see Mas-Hesse \& Kunth 1999).

In the following, we discuss the structural characteristics of the observed galaxies through an empirical classification scheme using morphological characteristics. A possible qualitative interpretation for each group is presented. From the velocity field structure we also examine the possible relationship between starburst triggering mechanisms and star formation.

\subsection{The continuum and the emission lines sructure of BCDGs: Possible clues to their star formation history}

There is now in the literature a bulk of observational evidence that the active star-forming regions that provide emission lines and high surface brightness in UV or blue spectral bands are hosted in a galaxy containing an old evolved stellar population whose light is dominated by red giant stars at visible wavelengths (Doublier et al. 1997, 1999, 1998; Papaderos et al. 1996; Telles et al. 1997; Kunth et al. 1988). Therefore, one observes, on the line of sight, a mixture of stellar components of various ages, the resulting color obviously depending on the star formation history of each object. Elaborate grids have been developed, by Olofson (1989), Copetti et al. (1986), Mas-Hesse \& Kunth (1993), Leitherer \& Heckman (1995) and other that can help understanding all these observed morphologies and associated spectroscopic characteristics.

In the following, we shall make the following assumptions, as a working hypothesis:

- [OIII] and $\mathrm{H} \alpha$ emission line peaks trace the sites where current or very recent (age less than $20 \mathrm{Myr}$ ) star formation takes place,

- a high excitation ratio $[\mathrm{OIII}] / \mathrm{H} \beta$ indicates the youngest (most massive) ionizing stars in these regions of current star formation, and should trace the most recent places of star formation,

- regions with blue and red continuum features correspond to older sites of star formation: the blue continuum trace star formation episodes with ages from $100 \mathrm{Myr}$ to 1-2 Gyr (mainsequence dominated stellar population components), while the red continuum is a better indicator of evolved post mainsequence stars.

We must underline that from the limited information of two intermediate (400 $\AA$ ) synthesized spectral bands images it is impossible to discriminate the presence of young red supergiants (supposing that the metallicity is high enough for such stars to appear) from a $1 \mathrm{Gyr}$ evolved population of intermediate mass stars or from a normal red giant dominated old population (5-10 Gyr). Let us recall that on a limited sample of 12 BCDGs, Doublier et al. 1999 have shown that a $K$-band excess was present in 11 objects, that provided evidence for a substantial population of asymptotic giants of intermediate mass. Such stars have anyway a much larger age than that of the observed current starburst in BCDGs.

The preceding simplified view is illustrated in several objects in which a fair positional coincidence is observed between the emission lines brightness maxima and the blue continuum 
peaks while the red continuum distribution has a different shape or location (e.g. Mrk 1499): in such cases, the blue continuum probably traces the position of the newborn massive super-star cluster responsible for the ionization of the HII region, while the red continuum morphology is representative of different, older stellar population components.

Turning now to the galaxies observed in the present work, we tentatively divide them in two subclasses:

a) Galaxies with a single star forming site, where all images morphologically coincide: Mrk 33, 826, 1434, 1450, 1480, SBS $0136+328$, SBS $1400+461$, SBS $1428+457$, and SBS $1632+579$. In the following, we shall refer to this subclass as "objects of type $a$ ". Our $a$ subclass is largely consistent with the $n E$ class of BCDGs as defined, from wide band imaging, by Loose \& Thuan (1986a).

We speculate that in these objects, the red continuum indicates the presence of a stellar population component significantly older than the one formed in the present phase of starforming activity (i.e. older than the red supergiants that could provide this red continuum). The justification for this is the tendency for the red continuum to slightly extend beyond the blue one. Supergiants stars tend to have a patchy distribution similar to the young blue massive stars. However since all images are coeval, we must accept the conclusion that the spatial location of the star forming activity has remained the same, whatsoever, bulding up the stellar density peak observed in the red. This has obvious dynamical implications. Indeed these BCDGs have a present-day star forming activity by unit mass much larger than ordinary giant galaxies. The starburst hypothesis is widely accepted since a continuous star formation at the observed rate would exhaust the expected available gas within a few tens of Myrs. Furthermore, the expected supernova and superwind activity in massive star forming complexes are likely to blow out the gas, that could not be available for a new starburst until it has cooled down sufficiently or has been replaced by newly accumulated gas through some efficient dynamical process.

Hence, either the presently observed star forming event is the first one occurring since the formation of the observed red "nucleus", or there has been several past such events in the history of the galaxy. In any case, the dynamical process that ensures the necessary accumulation of gas at the location of the same star-forming site must be a permanent feature in the dynamics of the object. It is useful to underline that the observed star-forming clumps in this class of objects have a general tendency to be centrally located with respect to the outer isophotes (i.e. Doublier et al. 1997). It is tempting to speculate that the gas accumulates at the bottom of the gravitational potential well.

b) Galaxies in which we observe a positional discrepancy between continuum and emission lines distributions (hereafter subclass $b$ ). We tentatively assume that these discrepancies are due to the spatial migration of the star forming sites over the history of the object. In this subclass we find: Mrk 324, 900, 1426, 1499, SBS 1154+534, SBS 1331+493, and SBS $1707+565$. The situations are various: some objects exhibiting only one single current massive star forming center, i.e. a single ionized gas component: e.g. Mrk 324, 900, 1499, SBS $1707+565$, among which there exist at least one galaxy with two continuum components, Mrk 324. The other galaxies exhibit two or more star forming centers (SBS 1331+493 is much more extended than the presently observed area and has several knots in emission, while we probably resolve three components in Mrk 1426).

In Mrk 900 and SBS 1331+493, the past and recent SF activity have taken place at identical locations but the current SF site (at least one) has migrated since the last active episode. In Mrk 324, 1426, SBS 1154+534 and SBS 1707+565, the respective locations of the past, recent and current star formation sites are all different, implying systematic spatial migration of the star-forming activity between each starburst phase.

Mrk 1499 is a special case: the blue and red continuum peaks are separated. The red continuum peak is offseted with respect to the center of the ionized gas component while the blue continuum one is nearly coincident with it.

c) Two objects deserve a separate discussion:

Although Mrk 1416 possesses a double component central structure, its brightest component suggests an $a$ subclass. Note however that the dynamical process in this case is probably much more intricate than for most subclass $a$ objects.

SBS $1723+565 B$ is a high luminosity galaxy but its structure is similar to that of a BCDG with double star forming sites. In each of them, spatial differences between components of various ages are evident, that provide similarities with the subclass $b$ dwarf galaxies as regards to the star formation history.

Objects of both groups, $a$ and $b$, provide excellent candidates for the star formation modeling in BCDGs. Galaxies from $b$ group are specially interesting to test the model of selfpropagating star formation (e.g. Gerola et al. 1980).

\subsection{The structures of BCDGs in [SII] emision lines}

We have suggested above that comparative mapping of the extended ionized regions in $\mathrm{H} \alpha$ and [SII] emission lines may provide indications on possible shock heating in these regions. The source of shock heating can be supernovae explosions ( $\mathrm{SNe}$; e.g. Morse et al. 1996), advanced merging in closely interacting galaxy pairs (e.g. Lamb et al. 1994; or superbubbles (e.g. Heckman 1995).

Morphological differences between [SII] lines intensity distributions and star formation zones traced by ionized hydrogen seen in $\mathrm{H} \alpha$ have been discovered in Mrk 324 and 1434 and are suspected also in Mrk 900. In Mrk 324 the [SII] lines exhibit an arc like structure located between two past and one recent star formation sites. This structure may reflect the existence of a shock heated gas component resulting from the interaction of outflowing gas from these sites of star formation. In Mrk 900 a similar situation is likely to occur. In Mrk 1434 the [SII] lines show a double component structure around the compact and highly concentrated peak of star formation. It can be interpreted as an evidence of existence of superbubble structure in this galaxy (see below). 
In the remaining 10 galaxies, the [SII] distributions do not exhibit morphological anomalies: if a shocked gas is present, its signature is not observable.

\subsection{Velocity fields}

The tentative classification outlined above, based on the continuum versus emission line morphologies, shows a large variety of cases, suggesting different evolution patterns of the star forming activity among BCDGs. We suspect that the triggering and dynamical mechanisms responsible for the fueling of the starburst could be different from one BCDG subclass to another: this is not in contradiction with earlier semi-empirical classifications (Loose \& Thuan 1986a) or with observations of photometric types that may imply vastly different overall dynamic properties of the objects (Kunth et al. 1988; Doublier et al. 1997, 1999; Papaderos et al. 1996). The properties of the velocity field observed in BCDGs may provide clues to answer these questions.

\subsubsection{Galaxies without ordered rotational pattern}

6 objects (Mrk 900, 1426, 1434, 1450, SBS 1154+534 and SBS $1632+579$ ) show a very disturbed or homogeneous velocity field. We recall that observed homogeneity may simply result from the lack of spatial and spectral resolution on a naturally chaotic velocity pattern. Hence, we prefer to use the word "chaotic" to describe these non-ordered motions. It is worth mentioning that among these 6 objects, 4 have been studied in wide band surface photometry and have revealed a brightness distribution dominated by an $r^{1 / 4}$ law, hence implying some degree of dynamical relaxation as regards to the stellar mass distribution. Among these 6 galaxies, Mrk 1450 has a very compact structure and belongs to subclass $a$ as defined above. Mrk 1434 also belongs to subclass $a$ from its emission line and continuum morphology recalling that the double-peak structure of the [SII] lines intensity distribution coincides with local maxima observed in the velocity map. This strengthens the superbubble hypothesis whose projected expansion velocity pattern could blur out any regular background pattern.

\subsubsection{Galaxies with ordered rotational pattern}

9 objects have a rather regular velocity field structure suggesting ordered circular rotational motions. They are Mrk 33, $324,826,1416,1499$, SBS $0136+328$, SBS 1400+461, SBS $1428+457$, SBS $1707+565$. The observed field on SBS $1331+493$ do not cover a sufficient angular area of the galaxy but ordered rotation on a large scale is not excluded. In Mrk 1480 a quite regular velocity gradient is seen on the observed southern half of the compact component, but we have no information on the Northern half.

Five galaxies: Mrk 33, Mrk 826, SBS 0136+328, SBS $1400+461$, SBS $1428+457$ are typical examples of subclass $a$ objects. In these BCGs, we probably observe either one single SF region, or possibly several (unresolved at our spatial resolution), highly concentrated SF clumps in which the ionized gas rotate as a single solid body (except in Mrk 826, see below). It is clear that in these galaxies, isovelocity contours are less disturbed than in galaxies with multiple star forming centres. Note however the large prevalence (4 objects out of 5) of a discrepancy between the kinematic line of nodes and the PA of the photometric major axis, indicating some dynamical decoupling between the gas and the stellar components.

The observed radial velocity gradients $(0.070$ to $0.118 \mathrm{~km} \mathrm{~s}^{-1} \mathrm{pc}^{-1}$ ) and spherical mass estimates (2.6 to $4 \times 10^{8} M_{\odot}$ ) inside radii from 480 to $690 \mathrm{pc}$, for Mrk 33, SBS $1400+461$ and SBS $1428+457$, are in agreement with data obtained for other BCDGs (Petrosian et al. 1997; van Zee et al. 1998). Mrk 826 presents a significantly higher mass concentration and hence a strong velocity gradient, but the elongated and distorted isovelocity lines suggest the presence of non-axisymmetric perturbations. SBS $0136+328$ is the second most luminous object in our sample, with a radial velocity gradient and a spherical mass value more typical of the larger luminosity BCGs studied by Östlin et al. (1999).

The remaining 4 galaxies (Mrk 324, 1416, 1499, SBS $1707+457$ ) are of subclass $b$. Deformations of the velocity contours, disordered motions superimposed over the regular velocity fields in all objects and large discrepancies between kinematic and photometric major axis orientations for 3 of 4 galaxies show that local dynamical instabilities and disturbances are common. These suggest that the gas is locally not in equilibrum, possibly because of gravitational effects of the multiple star-forming centres coupled with explosive events. The values of radial velocity gradients are in agreement with previous determinations (e.g. van Zee et al. 1998).

\subsubsection{SBS $1723+565 B$}

This galaxy has a regular velocity structure. Its absolute luminosity, morphology, velocity field structure and dynamical mass estimate make this galaxy very similar to the galaxy ESO 480-IG12, studied by Östlin et al. (1999).

Table 3 summarizes the observed properties and empirical classification of the 18 observed galaxies and is selfexplanatory. The sources for the photometric type for Mrk33 is Loose \& Thuan (1986b), and Doublier et al. (1997) for the remaining galaxies. Both articles describe the procedure for the photometric type determination together with the corresponding error analysis. The excitation pattern is "simple" when the excitation gradients observed on the $[\mathrm{OIII}] / \mathrm{H} \beta$ ratio map are consistent with the [OIII] brightness gradients, and "complex" if the excitation map shows unexpected features as secondary maxima or "ridges" that do not correspond spatially with the maxima in [OIII] line intensity.

\section{Conclusions and summary}

We report the results of an integral field multi-pupil spectroscopic study of a sample of 17 Blue Compact Dwarf Galaxies and the object SBS $1723+565 \mathrm{~B}$, selected from the First and Second Byurakan Surveys. An atlas of $\mathrm{H} \alpha$, [OIII] and [SII] emission lines, red and blue continuum, and $[\mathrm{OIII}] / \mathrm{H} \beta$ ratio maps has been produced. We also present velocity fields 
Table 3. Summary of the observed properties and empirical classification of the 18 observed galaxies.

\begin{tabular}{|c|c|c|c|c|c|c|c|}
\hline Galaxy & Subclass & $M_{B}$ & $\begin{array}{r}\text { Excitation } \\
\text { pattern }\end{array}$ & Photometric type & Gas motions & $\begin{array}{r}\text { Spherical mass } \\
\text { estimate }\end{array}$ & in radius \\
\hline Mrk33 & $a$ & -18.4 & simple & $r^{1 / 4}$ dominant & ordered & $4.0 \times 10^{8} M_{\odot}$ & $500 \mathrm{pc}$ \\
\hline Mrk324 & $b$ & -16.0 & complex & $r^{1 / 4}$ dominant & ordered & $0.57 \times 10^{8} M_{\odot}$ & $400 \mathrm{pc}$ \\
\hline Mrk826 & $a$ & -15.0 & complex & (unknown) & ordered & $2.0 \times 10^{8} M_{\odot}$ & $140 \mathrm{pc}$ \\
\hline Mrk900 & $b$ & -16.6 & complex & $r^{1 / 4}$ & chaotic & & \\
\hline Mrk1416 & $a$ & -15.4 & simple & exponential & ordered & $0.78 \times 10^{8} M_{\odot}$ & $370 \mathrm{pc}$ \\
\hline Mrk1426 & $b$ & -15.5 & complex & $r^{1 / 4}$ & chaotic & & \\
\hline Mrk1434 & $a$ & -15.9 & simple & $r^{1 / 4}$ dominant & chaotic & & \\
\hline Mrk1450 & $a$ & -14.6 & simple & $r^{1 / 4}$ & chaotic & & \\
\hline Mrk1480 & $a$ & -15.4 & simple? & $r^{1 / 4}$ & ordered? & & \\
\hline Mrk1499 & $b$ & -17.2 & simple, flat & non standard & ordered & $3.6 \times 10^{8} M_{\odot}$ & $490 \mathrm{pc}$ \\
\hline SBS0136+328 & $a$ & -16.6 & simple & $r^{1 / 4}$ dominant & ordered & $17 \times 10^{8} M_{\odot}$ & $1050 \mathrm{pc}$ \\
\hline SBS1154+534 & $b$ & -16.7 & complex & (unknown) & chaotic? & & \\
\hline SBS1331+493 & $b$ & -14.6 & complex & non standard & ? & & \\
\hline SBS1400+461 & $a$ & -15.8 & complex & (unknown) & ordered & $2.6 \times 10^{8} M_{\odot}$ & $480 \mathrm{pc}$ \\
\hline SBS1428+457 & $a$ & -16.5 & simple & exponential & ordered & $3.7 \times 10^{8} M_{\odot}$ & $690 \mathrm{pc}$ \\
\hline SBS1632+579 & $a$ & -15.2 & simple? & (unknown) & chaotic & & \\
\hline SBS1707+565 & $b$ & -16.3 & complex & (unknown) & ordered & $1.6 \times 10^{8} M_{\odot}$ & $980 \mathrm{pc}$ \\
\hline SBS1723+565B & $b$ & -19.7 & simple & (unknown) & ordered & $1.5 \times 10^{10} M_{\odot}$ & $2500 \mathrm{pc}$ \\
\hline
\end{tabular}

pertaining to the ionized gas component of the observed galaxies. The integrated spectroscopic properties of the studied star-forming regions are derived. From this atlas and velocity fields, we classify the objects in the following groups:

1) Ten objects have emission lines and continuum brightness distributions in fair spatial coincidence with each other. Further, the excitation parameter maps have also similar structure as intensity maps. One object among these 10 galaxies (Mrk 1416) has a double peaked structure. The remaining 9 galaxies show a single conspicuous peaked compact structure. We suggest that these galaxies are objects experiencing continuous (or repeating bursts of) star formation at the same spatial location, implying that the dynamical process that feeds the stellar formation with gas is stable over long timescales.

2) Eight objects have emission lines, excitation parameter and continuum distributions that differ from each other. These objects show one, double or multiple components structure in emission lines and/or in continuum as well as complex structure in their excitation parameter distribution.

Several observed features may be linked to recent past interactions or ongoing merging phenomena.

It is also possible that these galaxies are objects with different age and different local star formation events.

3) One object (Mrk 1434) whose [SII] brightness distribution differs from that observed in the [OIII] and Balmer lines as well as in the blue and red images. This galaxy shows evidence for the existence of a superbubble structure or a $\mathrm{SNe}$ event in its SF region. Superbubble structures or SNe events could further explain the observed [SII] lines emitting morphologies in 2 other objects of the sample.

4) Ten galaxies show quite regular radial velocity gradient, globally consistent with the solid-body-like rotation of a disk of ionized gas. However, the kinematical major axis position angle of the ionized gas in 8 objects is in strong discrepancy with the value of the position angle of the photometric major axis. This could be due to dynamical disturbances and instabilities. A detailed modelling of the internal dynamics of such small angular extent objects is difficult from the present results and would need more observations at a substantially better angular resolution.

5) Six objects have either irregular and distorted or nearly homogeneous ("chaotic") velocity fields, implying that even if the objects are supported by rotation, the kinematic signature of this rotation is strongly perturbed by non-axisymmetric phenomena across the central regions. The disturbed structure of the velocity field of Mrk 1434 can originate from an expanding superbubble.

For Mrk 1480 the present observational material appears insufficient to derive a firm conclusion about the velocity field structure.

6) SBS $1723+565 B$ from its blue absolute luminosity $\left(M_{B}=-19.7\right)$, morphology, radial velocity field structure, and dynamical mass value is similar to the giant, star-forming irregular galaxies. Because it shows an obvious double component structure, it can be an interacting pair of galaxies in an advanced phase of merging.

Acknowledgements. This work was partly supported by the Jumelage Astrophysique France-Arménie a bilateral program funded by Centre National de la Recherche Scientifique, the French Ministère des Affaires Etrangères and Ministère de la Recherche et de la Technologie. The financial support of INTAS through Project 93-2032 and 93-2032-Extension is gratefully acknowledged. This research has made use of the NASA/IPAC Extragalactic Database (NED) which is operated by the Jet Propulsion Laboratory, California Institute of Technology, under contract with the NASA. The SAO 6 m telescope Time Allocation Comittee is gratefully thanked for generous grant of 
observing time. The technical staff of SAO has provided invaluable help in instrument setting and software assistance.

\section{References}

Abrahamian, H. V., \& Saakian, K. A. 1989, Afz, 31, 447

Afanasiev, V. L., Vlasyuk, V. V., Dodonov, S. N., \& Sil'chenko, O. K. 1990, SAO prepr. No. 54

Arkhipova, V. P., Noskova, R. I., Sil'chenko, O. K., \& Zasov, A. V. 1987, SvALett, 13, 240

Bacon, R., Adam, G., Baranne, A., et al. 1995, A\&AS, 113, 347

Barbieri, C., Bonoli, C., \& Rafanelli, P. 1979, A\&AS, 37, 541

Brinks, E. 1990, in Dynamics and Interactions of Galaxies, ed. R. Wielen (Berlin: Springer), 146

Combes, F. 1994, in Star formation, galaxies and the interstellar medium, ed. J. Franco, F. Ferrini, \& G. Tenorio-Tagle (Cambridge Univ. Press: Cambridge), 324

Comte, G., Petrosian, A. R., Ohanian, G. A., \& Stepanian, J. A. 1999, Astrophys., 42, 149

Copetti, M. V. F., Pastoriza, M. G., \& Dottori, H. A. 1986, A\&A, 156, 11

Courtès, G. 1982, in Instrumentation for Astronomy with Large Optical Telescopes, ed. C. M. Humphries (Reidel), 123

Doublier, V., Comte, G., Petrosian, A. R., Surace, C., \& Turatto, M. 1997, A\&AS, 124, 405

Doublier, V., Caulet, A., \& Comte, G. 1998, in Dwarf Galaxies and Cosmology, proceedings of the XXXIIIrd Rencontres de Moriond, ed. by T. X. Thuan, C. Balkowski, V. Cayatte \& J. Tran Thanh Van (Éditions Frontières), 215

Doublier, V., Caulet, A., \& Comte, G. 1999, A\&AS, 138, 213

Edmunds, M. G., \& Pagel, B. E. J. 1984, MNRAS, 211, 507

Erickson, L. K., Gottesman, S. T., \& Hunter, Jr., J. H. 1999, ApJ, 515, 153

Evans, I. N., \& Dopita, M. A. 1985, ApJS, 58, 125

Gallagher, III J. S., \& Hunter, D. A. 1983, ApJ, 274, 141

Gallagher, III J. S., \& Hunter, D. A. 1989, AJ, 98, 806

Gallagher, III J. S., Hunter, D. A., \& Tutukov, A. V. 1984, ApJ, 284, 544

Gerola, H., Seiden, P. E., \& Schulman, L. S. 1980, ApJ, 242, 517

Güsten, R., \& Mezger, P. 1983, Vistas in Astron., 26, 159

Heckman, T. 1995, in The interplay between massive star formation, the ISM and galaxy evolution, ed. D. Kunth, B. Guiderdoni, \& T. X. Thuan (Éditions Frontières), 159

Hunter, D. A., \& Gallagher, III J. S. 1986, PASP, 98, 5

Hunter, D. A., \& Thronson, Jr. H. A. 1995, ApJ, 452, 238

Izotov, Y. I., Thuan, T. X., \& Lipovetsky, V. A. 1994, ApJ, 435, 647

Izotov, Y. I., Thuan, T. X., \& Lipovetsky, V. A. 1997, ApJS, 108, 1

Kennicutt, Jr. R. C. 1992, ApJ, 388, 310

Kennicutt, Jr. R. C., Keel, W. C., van der Hulst, J. M., Hummel, E., \& Roettinger, K. A. 1987, AJ, 93, 1011

Korovyakovskii, Y. P., Petrosian, A. R., Saakian, K. A., \& Khachikian, E. E. 1981, Afz, 17, 231

Kunth, D., Maurogordato, S., \& Vigroux, L. 1988, A\&A, 204, 10

Lamb, S. A., Gerber, R. A., \& Balsara, D. S. 1994, in Violent star formation from 30 Doradus to QSOs, ed. G. Tenorio-Tagle (Cambridge Univ. Press, Cambridge), 243

Legrand, F. 2000, A\&A, 354, 504

Legrand, F., Kunth, D., \& Mas-Hesse, J. M. 1997, A\&A, 326, 929

Legrand, F., Kunth, D., Roy, J.-R., Mas-Hesse, J. M., \& Walsh, J. R. 2000, A\&A, 355, 891

Leitherer, C., \& Heckman, T. M. 1995, ApJS, 96, 9
Lequeux, J. 1980, in Star Formation, ed. A. Maeder, \& L. Martinet (Sauverny: Geneva Observatory), 77

Lequeux, J., Kunth, D., Mas-Hess, J. M., \& Sargent, W. L. W. 1995, A\&A, 301, 18

Li, F., \& Ikeuchi, S. 1989, PASJ, 41, 221

Loose, H. H., \& Thuan, T. X. 1986a, in Star Forming Dwarf Galaxies, ed. D. Kunth, T. X. Thuan, \& J. T. T. Van (Éditions Frontières), 73

Loose, H. H., \& Thuan, T. X. 1986b, ApJ, 309, 59

Marconi, G., Matteucci, F., \& Tosi, M. 1994, MNRAS, 270, 35

Martin, C. 1996, ApJ, 465, 680

Mas-Hesse, J. M., \& Kunth, D. 1993, A\&AS, 88, 399

Mas-Hesse, J. M., \& Kunth, D. 1999, A\&A, 349, 765

Massey, P., Strobel, K., Jeannette, V., Anderson, B., \& Anderson, E. 1988, ApJ, 328, 315

Matteucci, F., \& Tosi, M. 1985, MNRAS, 217, 391

Mazzarella, J., \& Boroson, T. 1993, ApJS, 85, 27

Moles, M., Garcia-Pelayo, J. M., del Rio, G., \& Lahulla, F. 1987, A\&A, 186, 77

Morse, J. A., Raymond, J. C., \& Wilson, A. S. 1996, PASP, 108, 426

Munari, U., Zwitter, T., \& Mikuz, H. 1995, A\&A, 296, 310

Noguchi, M. 1991, MNRAS, 251, 360

Olofsson, K. 1989, A\&AS, 80, 317

Östlin, G., Amram, Ph., Masegosa, J., Bergvall, N., \& Boulesteix, J. 1999, A\&AS, 137, 419

Östlin, G., \& Kunth, D. 2001, A\&A, 371, 429

Pagel, B. E. J., Edmunds, M. G., \& Smith, G. 1980, MNRAS, 193, 219

Papaderos, P., Loose, H. H., Thuan, T. X., \& Fricke, K. J. 1996, A\&AS, 120, 207

Petrosian, A. R., Boulesteix, J., Comte, G., Kunth, D., \& Le Coarer, E. 1997, A\&A, 318, 390

Pustilnik, S. A., Ugryumov, A. V., Lipovetsky, V. A., Thuan, T. X., \& Guseva, N. G. 1995, ApJ, 443, 499

Roy, J.-R., \& Kunth, D. 1995, A\&A, 294, 432

Roy, J.-R., Belley, J., Dutil, Y., \& Martin, P. 1996, ApJ, 460, 284

Saito, M., Sasaki, M., Ohta, K., \& Yamada, T. 1992, PASJ, 44, 593

Sanders, R. H., \& Verheijen, M. A. W. 1998, ApJ, 503, 97

Schwarts, R. D., Cohen, M., Jones, B. F., et al. 1993, AJ, 106, 740

Searle, L., \& Sargent, W. L. W. 1972, ApJ, 173, 25

Shull, J. M., \& McKee, C. F. 1979, ApJ, 227, 131

Sil'chenko, O. K. 1997, Bull. Spac. Astrophys. Obs., 44, 30

Silk, J. 1995, ApJ, 438, L41

Skillman, E. D., \& Kennicutt, Jr. R. C. 1993, ApJ, 411, 655

Telles, E., Melnick, J., \& Terlevich, R. 1997, MNRAS, 288, 78

Terlevich, R., \& Melnick, J. 1981, MNRAS, 195, 839

Thronson, Jr. H. A., \& Telesco, C. M. 1986, ApJ, 311, 98

Thuan, T. X., \& Martin, G. E. 1981, ApJ, 247, 823

Thuan, T. X., Williams, T. B., \& Malumuth, E. 1987 in Star Bursts and Galaxy Evolution, ed. T. Montmerle, T. X. Thuan, \& T. T. Van (Éditions Frontières), 151

Thuan, T. X., Izotov, Y. I., \& Lipovetsky, V. A. 1995, ApJ, 445, 108

Thuan, T. X., Lipovetsky, V. A., Martin, J.-M., \& Pustilnik, S. A. 1999, A\&AS, 139, 1

Tomita, A., Ohta, K., \& Saito, M. 1993, PASJ, 45, 693

van Zee, L., Skillman, E. D., \& Salzer, J. J. 1998, AJ, 116, 1186

Viallefond, F., Lequeux, J., \& Comte, G. 1987, in Starbursts and Galaxy Evolution, ed. T. X. Thuan, T. Montmerle, \& J. T. Van (Éditions Frontières), 139

Vlasiuk, V. V. 1993, Izv. SAO, 36, 107

Zaritsky, D. 1992, ApJ, 390, L73 